\title{
1 Gradients in the cerebellar cortex enable Fourier-like transformation and 2 improve storing capacity
}

3 Isabelle Straub ${ }^{1^{*}}$, Laurens Witter ${ }^{1,2^{*}}$, Abdelmoneim Eshra ${ }^{1^{*}}$, Miriam Hoidis ${ }^{1^{*}}$, 4 Niklas Byczkowicz ${ }^{1}$, Sebastian Maaß ${ }^{1}$, Igor Delvendahl ${ }^{1,3}$, Kevin Dorgans ${ }^{4}$, Elise 5 Savier $^{4}$, Ingo Bechmann ${ }^{5}$, Jens Eilers ${ }^{1}$, Martin Krüger $^{5}$, Philippe Isope ${ }^{4}$, and 6 Stefan Hallermann ${ }^{1, \#}$

$7 \quad{ }^{1}$ Carl-Ludwig-Institute for Physiology, Medical Faculty, University of Leipzig, Liebigstrasse 27, 804103 Leipzig, Germany

92 Department of Integrative Neurophysiology, Center for Neurogenomics and Cognitive Research 10 (CNCR), VU University, De Boelelaan 1085, 1081 HV Amsterdam, The Netherlands

$11{ }^{3}$ current address: Institute of Molecular Life Sciences, University of Zurich, Winterthurerstrasse 12190,8057 Zurich, Switzerland

$13{ }^{4}$ Institut des Neurosciences Cellulaires et Intégratives, CNRS, Université de Strasbourg, 8, allée 14 du Général Rouvillois, 67084 Strasbourg, France

$15{ }^{5}$ Institute of Anatomy, Medical Faculty, University of Leipzig, Leipzig, Germany

16 * These authors contributed equally to this work

17 " Corresponding author

\section{Abstract}

21 Cerebellar granule cells (GCs) making up majority of all the neurons in the 22 vertebrate brain, but heterogeneities among GCs and potential functional 23 consequences are poorly understood. Here, we identified unexpected gradients 24 in the biophysical properties of GCs. GCs closer to the white matter (inner-zone 25 GCs) had higher firing thresholds and could sustain firing with larger current 26 inputs. Dynamic clamp experiments showed that inner- and outer-zone GCs 27 preferentially respond to high- and low-frequency mossy fiber inputs, 28 respectively, enabling to disperse the mossy fiber input into its frequency 29 components as performed by a Fourier transformation. Furthermore, inner-zone 30 GCs have faster axonal conduction velocity and elicit faster synaptic potentials in 31 Purkinje cells. Neuronal network modeling revealed that these gradients improve 32 spike-timing precision of Purkinje cells and decrease the number of GCs required 33 to learn spike-sequences. Thus, our study uncovers biophysical gradients in the 34 cerebellar cortex enabling a Fourier-like transformation of mossy fiber inputs. 


\section{Introduction}

36 Digital audio compression (e.g., 'MP3'; Jayant et al., 1993) and image 37 compression (e.g., 'JPEG'; Wallace, 1992) rely on Fourier transformations, which

38 decompose a signal (e.g., sound amplitude as a function of time or image 39 intensity as a function of space) into its frequency components (power as a 40 function of frequency). By storing these frequency components with different 41 precision depending on psychophysical demands of hearing and seeing, the 42 overall storage capacity can be increased dramatically. In principle, neuronal 43 networks consisting of neurons with varied electrophysiological properties could 44 be suitable for Fourier-like transformations of information. This could benefit 45 processing in neuronal circuits by increasing the signal-to-noise ratio of input 46 signals or by selecting only relevant spectral components of a signal.

47 Interestingly, there are indications that for example pyramidal neurons in visual 48 cortex and in the hippocampus are tuned to different inputs or different input 49 strengths (Cembrowski and Spruston, 2019; Fletcher and Williams, 2019; Soltesz 50 and Losonczy, 2018). However, whether these neuronal networks perform a 51 Fourier-like transform on their inputs remains unknown.

52 Controlling the timing and precision of movements is considered to be one of the 53 main functions of the cerebellum. In the cerebellum, the firing frequency of 54 Purkinje cells (PCs) (Heiney et al., 2014; Herzfeld et al., 2015; Hewitt et al., 55 2011; Medina and Lisberger, 2007; Payne et al., 2019; Sarnaik and Raman, 56 2018; Witter et al., 2013) or the timing of spikes (Brown and Raman, 2018; 57 Sarnaik and Raman, 2018) have been shown to be closely related to movement. 58 Indeed, cerebellar pathology impairs precision in motor learning tasks (Gibo et 59 al., 2013; Martin et al., 1996) and timing of rhythmic learning tasks (Keele and 60 Ivry, 1990). These functions are executed by a remarkably simple neuronal 61 network architecture. Inputs from mossy fibers (MFs) are processed by GCs and 62 transmitted via their parallel fiber (PF) axons to PCs, which provide the sole 63 output from the cerebellar cortex. GCs represent the first stage in cerebellar 64 processing and have been proposed to provide pattern separation and 
65 conversion into a sparser representation of the MF input (recently reviewed by 66 (Cayco-Gajic and Silver, 2019). These MF inputs show a wide variety of signaling 67 frequencies, ranging from slow modulating activity to kilohertz bursts of activity 68 (Arenz et al., 2008; Rancz et al., 2007; Ritzau-Jost et al., 2014; van Kan et al., 69 1993). Interestingly, in cellular models of the cerebellum, each MF is considered 70 to be either active or inactive with little consideration for this wide range of 71 frequencies (Albus, 1971; Marr, 1969). Furthermore, in these models, GCs are

72 generally considered as a uniform population of neurons.

73 Here we show that the biophysical properties of GCs differ according to their 74 vertical position in the GC layer. GCs located close to the white matter (inner75 zone) selectively transmit high-frequency MF inputs, have shorter action 76 potentials and a higher voltage threshold to fire an action potential compared with 77 GCs close to the PC layer (outer-zone). This gradient of GC properties enables a 78 Fourier-like transformation of the MF input, where inner-zone GCs convey the 79 high-frequency and outer-zone GCs the low-frequency components of the MF 80 input. These different Fourier-like components are sent to PCs by specialized 81 downstream signaling pathways, differing in PF axon diameters, action potential 82 velocity and PC excitatory postsynaptic potential (EPSP) kinetics. Computational 83 simulations show that the biophysical gradients in the GC and molecular layer

84 significantly reduce the number of GCs required to learn a sequence of firing 85 frequencies and accelerate the time required to switch between firing 86 frequencies.

\section{Results}

89 A gradient in the biophysical properties of inner- to outer-zone GCs

90 To investigate whether GCs are tuned for different frequencies we first 91 investigated the intrinsic membrane properties of GCs from different depths 92 within the GC layer in lobule $V$ of the cerebellum of P21-30 mice. We divided the 93 GC layer into three zones and performed whole-cell current-clamp recordings 
94 from inner- (closest to the white matter), middle- and outer-zone (closest to PCs) 95 GCs (Figure1A,B). Upon current injection, inner-zone GCs were less excitable 96 compared with outer-zone GCs (Figure 1C). On average, the relationship 97 between the mean number of action potentials and the injected current were 98 surprisingly different for inner- and outer-zone GCs (Figure 1D): inner-zone GCs 99 needed higher current injections to fire an action potential (inner: $56.8 \pm 2.6 \mathrm{pA}$ 100 vs. middle: $51.2 \pm 2.0 \mathrm{pA}$ vs. outer: $39.4 \pm 2.0 \mathrm{pA} ; \mathrm{n}=38,31$, and 38 , 101 respectively; $P_{A N O V A}<0.0001$; Figure $1 E$ ) and to achieve the maximum firing rate 102 compared with middle- and outer-zone GCs (inner: $224.6 \pm 9.8$ pA vs. middle: $103190.8 \pm 9.6$ pA vs. outer: $174.3 \pm 9.0$ pA, respectively; $P_{\mathrm{ANOVA}}=0.0007$; Figure 104 1F). Consistently, inner-zone GCs had a more depolarized threshold for action 105 potential generation compared with middle- and outer-zone GCs $(-38.0 \pm 0.7 \mathrm{mV}$ 106 vs. $-38.2 \pm 0.8 \mathrm{mV}$ vs. $-41.4 \pm 0.6 \mathrm{mV} ; P_{\mathrm{ANOVA}}=0.001$; Figure $\left.1 \mathrm{G}\right)$ and a lower 107 input resistance $\left(486 \pm 27 \mathrm{M} \Omega\right.$ vs. $494 \pm 27 \mathrm{M} \Omega$ vs. $791 \pm 63 \mathrm{M} \Omega$; $\mathrm{P}_{\mathrm{ANOVA}}=$ $108<0.0001$; Figure 1H). Furthermore, the capacitance of inner-zone GCs was 109 significantly larger compared to the outer-zone GCs (inner: $5.8 \pm 0.2 \mathrm{pF}$ vs. 110 middle: $5.8 \pm 0.2 \mathrm{pF}$ vs outer: $4.6 \pm 0.1 \mathrm{pF} ; \mathrm{P}_{\mathrm{ANOVA}}=<0.0001$ Figure 1I). In 111 agreement with these findings we observed depolarization block in inner-zone 112 GCs at higher current inputs than for outer-zone GCs (Figure 1C,D). 113 Furthermore, a larger delay of the first spike was observed in inner- compared 114 with outer-zone GCs $\left(P_{T-T e s t}=0.01\right.$; Figure 1J; $P_{\text {ANOVA }}=0.0001$; Figure $\left.1 \mathrm{~K}\right)$. The 115 delay with $60 \mathrm{pA}$ current injection was $48 \pm 6 \mathrm{~ms}$ for inner-, $38 \pm 4 \mathrm{~ms}$ for middle-, 116 and $23 \pm 2$ ms for outer-zone GCs ( $n=32,25$, and 37 , respectively; note that 6 117 out of 38 inner-zone GC did not fire an action potential at $60 \mathrm{pA}$ ). Finally, the 118 action potential half-width of GCs differed significantly between the three zones 119 (inner: $122 \pm 2 \mu$ s vs. middle: $137 \pm 4 \mu \mathrm{s}$ vs. outer: $143 \pm 4 \mu \mathrm{s} ; \mathrm{P}_{\mathrm{ANOVA}}=0.0001$; 120 Figure 1L).

121 To test whether these gradients are specific to lobule $V$, we investigated GCs in 122 lobule IX. Here, we observed very similar gradients to lobule V (Figure 1-figure 123 supplement 1). In short, outer-zone GCs were more excitable and had broader 124 spikes compared with inner-zone GCs. Interestingly, the absolute values 
125 between lobule $\mathrm{V}$ and IX differed (Figure 1-figure supplement 1), consistent

126 with previously described differences in, e.g., the firing frequency in vivo between

127 these two lobules (Witter and De Zeeuw, 2015a; Zhou et al., 2014) and in the

128 differential density of Kv4 and Cav3 channel expression in GCs across different

129 lobules (Heath et al., 2014; Rizwan et al., 2016; Serôdio and Rudy, 1998). Taking

130 the large functional difference between spino- and vestibulo-cerebellum into

131 account (Witter and De Zeeuw, 2015b), these data suggest that different

132 biophysical properties of GCs is likely a conserved mechanism throughout the

133 entire cerebellar cortex, potentially tuning GCs to different frequencies.

134 Development can have large effects on the physiology of neurons, and GCs in 135 particular undergo profound changes during development (Dhar et al., 2018; 136 Lackey et al., 2018). To exclude confounding effects of developmental stage, we 137 tested whether these gradients were also present at a later developmental stage. 138 Recordings obtained from GCs in lobule $V$ in animals between 80 and 100 days 139 of age showed the very similar gradients as in young animals (Figure 1-figure 140 supplement 2). Together, these data show a prominent gradient in the 141 electrophysiological properties of GCs over the depth of the granule cell layer, 142 and that this gradient can consistently be found in different lobules and ages.

\section{Voltage-gated potassium currents are larger at inner-zone GCs}

144 To investigate possible causes for the gradient in the biophysical properties, we 145 investigated voltage-gated potassium (Kv) currents by performing voltage-clamp 146 recordings in outside-out patches from somata of inner- and outer-zone GCs in 147 lobule $\mathrm{V}$ (Figure 2A). The maximum Kv current was significantly higher in inner148 zone GCs $(282 \pm 29$ pA, $n=48)$ compared with outer-zone GCs $(221 \pm 28$ pA, $n$ $149=54, \mathrm{P}_{\text {Mann-Whitney }}=0.02$; Figure 2B). Neither the steady-state activation curve 150 (Figure 2C) nor the degree of inactivation (Figure 2D) was different between the 151 two GC populations. Furthermore, steady-state inactivation, which was 152 investigated with different holding potentials, was similar between inner- and 153 outer-zone GCs (Figure 2-figure supplement 1). These data suggest that 154 inner- and outer-zone GCs have a similar composition of Kv channels, but inner- 
155 zone GCs have a higher Kv channel density. The here observed larger Kv 156 currents in inner-zone GCs are consistent with the short action potential duration 157 of inner-zone GCs (cf. Figure 1). Thus, our data provide a biophysical 158 explanation for the observed gradient in GC properties.

\section{MF inputs are differentially processed by inner and outer GCs}

160 The gradient within the GC layer creates an optimal range of input strengths for 161 each GC. To test how this gradient impacts the processing of synaptic MF inputs, 162 we performed dynamic clamp experiments to test whether different MF input 163 frequencies differentially affect spiking in inner- and outer-zone GCs (Figure 3A). 164 We first recorded excitatory postsynaptic currents (EPSC) from GCs located at 165 inner- or outer-zone of lobule $V$ after single MF stimulation. We found no 166 significant differences in the amplitude nor in the kinetics of EPSCs in inner- and 167 outer-zone GCs (Figure 3-figure supplement 1).

168 Individual MFs span the entire depth of the GC layer, contacting both inner- and 169 outer-zone GCs (Krieger et al., 1985; Palay and Chan-Palay, 1974). 170 Furthermore, GCs are electronically extremely compact neurons and can be 171 considered as a single compartment (D'Angelo et al., 1993; Delvendahl et al., 172 2015; Silver et al., 1992). Therefore, we could use the dynamic clamp technique 173 to implement the conductance of identical MF signals in inner- and outer-zone 174 GCs based on the measured EPSC kinetics. We first applied input of a single MF 175 with Poisson-distributed firing-frequencies ranging between 30 and $500 \mathrm{~Hz}$ for $176300 \mathrm{~ms}$ duration while changing the resting membrane potential to simulate the 177 large variability of membrane potential of GCs observed in vivo (Chadderton et 178 al., 2004). In line with the observed gradients in the electrophysiological 179 properties of GCs, inner-zone GCs fired fewer action potentials compared with 180 outer-zone GCs in response to low-frequency MF inputs at a membrane potential 181 of $\sim 90 \mathrm{mV}$ (Figure 3B,C). In contrast, inner-zone GCs fired more action 182 potentials compared with outer-zone GCs in response to high-frequency MF 183 inputs at a membrane potential of $\sim-70 \mathrm{mV}$. In vivo, such a depolarization would 184 be caused by less inhibition and/or additional MF inputs. These data suggest that 
185 outer- and inner-zone GCs are specialized for low- and high-frequency MF 186 inputs, respectively.

\section{Fourier-like transformation of MF input frequency}

188 To further test whether inner- and outer-zone GCs can extract different frequency 189 components from a MF input signal, which would resemble a Fourier190 transformation, we varied the MF input frequency sinusoidally between 30 and $191300 \mathrm{~Hz}$ (Figure 4A). At a holding potential of $-70 \mathrm{mV}$, inner-zone GCs responded 192 preferentially to high-frequency MF inputs up to $300 \mathrm{~Hz}$, while outer-zone GCs 193 responded preferentially to low-frequency inputs up to $100 \mathrm{~Hz}$. To estimate the 194 optimal frequency at which inner- and outer-zone GCs preferentially fire action 195 potentials, we calculated the phase angle (see Methods). The mean phase 196 angle, at which GC preferentially fired, was $145.9 \pm 10.4^{\circ}$ for inner-zone $(n=13)$ 197 and $102.5 \pm 18.3^{\circ}$ for outer-zone GCs $(n=9)\left(P_{\text {T-TEST }}=0.04\right)$, representing an 198 average firing frequency of 217 and $100 \mathrm{~Hz}$ for inner- and outer-zone GCs, 199 respectively. Thus, the gradient in the biophysical properties enables the 200 cerebellar GC layer to split incoming MF signals into different frequency bands 201 and thereby to perform a Fourier-like transformation of the compound MF input 202 signal.

\section{The position of PFs is correlated with the position of GC somata}

204 A Fourier-like transformation in the GC layer (i.e. a separation of the spectral 205 components of MF signals) could be particularly relevant if downstream 206 pathways are specialized for these spectral components. Early silver-stainings 207 and drawings from Ramón y Cajal indicate that inner-zone GCs give rise to PFs 208 close to the PC layer and outer-zone GCs give rise to PFs close to the pia 209 (Eccles, 1967; Ramón y Cajal, 1911 but see Espinosa and Luo, 2008; Wilms and 210 Häusser, 2015). To test this possibility, we examined the ascending and parallel 211 branches of the GC axon. First, we investigated whether there is a correlation 212 between the relative positions of the PF in the molecular layer and the GC 213 somata in the GC layer. Dil was injected in vivo into the GC layer to label GCs 214 and their axons. Several GCs were clearly stained $24 \mathrm{~h}$ after Dil injection (Figure 
215 5A), and the position of their soma and PF in the cerebellar cortex could be 216 measured (Figure 5B-D). Even though the length of the ascending GC axon 217 showed considerable variation (196 $\pm 5.5 \mu \mathrm{m}$, range: 144 to $291 \mu \mathrm{m}, \mathrm{n}=39$ 218 axons in $\mathrm{n}=6$ mice), after normalization for the thickness of the molecular and 219 GC layers, GC soma position was significantly correlated with the position of the 220 bifurcation in the GC axon (Figure 5C,D; $R^{2}=0.86, P<0.001$ ). These data show 221 that inner- and outer-zone GCs preferentially give rise to inner- and outer-zone 222 PFs, respectively.

223 Inner-zone PFs have larger diameter and higher action potential 224 propagation velocity

225 Next, we tested whether PFs, like GCs, have different properties depending on 226 the position within the molecular layer. First, we compared the PF diameters in 227 electron microscopic images of parasagittal sections of mouse cerebellum lobule $228 \mathrm{~V}$ and found significantly larger diameters for inner-zone PFs compared to 229 middle- and outer-zone PFs (182 $\pm 2.6 \mathrm{~nm}, \mathrm{n}=703$ vs. $159 \pm 2.0 \mathrm{~nm}, \mathrm{n}=819$ vs.

$230145 \pm 1.7 \mathrm{~nm}, \mathrm{n}=1085$ Figure 6A-C; $P_{A N O V A}<0.0001$ ), which is in agreement 231 with previous investigations reported in cat (Eccles et al., 1967), monkey (Fox 232 and Barnard, 1957), and rats (Pichitpornchai et al., 1994).

233 Axonal diameter is often a strong predictor for axonal conduction speed (Jack et 234 al., 1983). We therefore recorded compound action potentials of PFs in lobule $V$ 235 and compared their conduction speed in the inner-, middle- and outer-zone of the 236 molecular layer (Figure 6D-F). We detected a significantly higher velocity in 237 inner-zone PFs compared with in middle- or outer-zone PFs $\left(0.33 \pm 0.004 \mathrm{~m} \mathrm{~s}^{-1}\right.$, $238 \mathrm{n}=8$ vs. $0.31 \pm 0.005 \mathrm{~m} \mathrm{~s}^{-1}, \mathrm{n}=6$ vs. $0.28 \pm 0.005 \mathrm{~m} \mathrm{~s}^{-1}, \mathrm{n}=8$; Figure $6 \mathrm{~F}$; $\left.239 \mathrm{P}_{\text {ANOVA }}<0.0001\right)$. The absolute velocity and the gradient in the velocity from 240 inner- to outer-zone PFs agree well with previous studies (Baginskas et al., 2009; 241 Vranesic et al., 1994). These results suggest that the inner-zone PFs are 242 specialized for fast signaling, which is consistent with the concept that inner-zone 243 GCs are tuned for high-frequency inputs (cf. Figure 1 and 2). 
244 In addition to the above results obtained from lobule $\mathrm{V}$, similar gradients in both

245 axon diameter and axon conduction speed were found in lobule IX (Figure 6-

246 figure supplement 1 ). This suggests that gradients in axon diameter and axon

247 conduction speed are general features of the cerebellar cortex.

248 A possible confounder of our results could be an overrepresentation of large249 diameter Lugaro cell axons within inner-zone PFs (Dieudonne and Dumoulin, 250 2000). However, this would predict that the histogram of the axon diameters 251 shows two distinct peaks with varying amplitude. Instead, we observed a single 252 bell-shaped distribution in each PF zone (Figure 6-figure supplement 2), 253 arguing that the measured differences between axon diameters were not due to 254 varying contributions from Lugaro cell axons, but reflect the differences between 255 inner-, middle- and outer-zone PFs.

\section{PCs process inner-, middle-, and outer-zone PF inputs differentially}

257 Our data thus far indicate that GCs and PFs are adapted to different MF input 258 frequencies and conduction velocity, respectively. This arrangement could in 259 principle provide PFs with functionally segregated information streams that are 260 differentially processed in PCs. To investigate this possibility, we made whole261 cell current-clamp recordings from PCs in sagittal slices of the cerebellar vermis.

262 PCs were held at a hyperpolarized voltage to prevent spiking and to isolate 263 excitatory inputs. Electrical stimulation of the PFs was alternated between inner-, 264 middle- and outer-zones and adjusted to obtain similar amplitude EPSPs in all 265 zones (Figure 7A,B). Stimulation of inner-zone PFs resulted in EPSPs (Barbour, 266 1993; Roth and Häusser, 2001) with shorter rise and decay times compared with 267 EPSPs obtained from stimulating outer-zone PFs (rise $20-80$ : inner: $0.57 \pm 0.04 \mathrm{~ms}$, $268 \mathrm{n}=12$; middle: $0.93 \pm 0.17 \mathrm{~ms}, \mathrm{n}=4$; outer: $1.83 \pm 0.33 \mathrm{~ms}, \mathrm{n}=12\left(\mathrm{P}_{\mathrm{ANOVA}}=\right.$ 269 0.009; decay: inner: $21.9 \pm 1.5 \mathrm{~ms}$, middle: $39.7 \pm 1.1 \mathrm{~ms}$ outer: $40.8 \pm 4.1 \mathrm{~ms}$; $270 \mathrm{P}_{\text {ANOVA }}=0.0004$, Figure 7C). These results suggest that inner-zone PF inputs 271 undergo less dendritic filtering in PCs compared with outer-zone PF inputs (De 272 Schutter and Bower, 1994a, b; Roth and Häusser, 2001) but see (De Schutter 273 and Bower, 1994c). To investigate high-frequency inputs to PCs, we elicited five 
274 EPSPs at $100 \mathrm{~Hz}$ and $500 \mathrm{~Hz}$ (Figure 7D,E). Individual EPSPs evoked from

275 inner-zone PFs showed clear individual rising phases and peaks between each

276 stimulus and less summation compared with outer-zone PFs (Figure 7D-F).

277 These results suggest that inner-zone PFs can transmit timing information more

278 faithfully compared with outer-zone PFs and thus control spike timing of PCs

279 more precisely.

280 The observed neuronal gradients increase storing capacity and improve 281 temporal precision of PC spiking

282 Thus far we have described a prominent gradient in the electrophysiological

283 properties of GCs over the depth of the GC layer that enables inner- and outer-

284 zone GCs to preferentially respond to high- and low-frequency inputs,

285 respectively. The different frequency components are transferred via specialized

286 PFs, which enable PCs to interpret high-frequency signals rapidly at the base of

287 their dendritic trees and low-frequency signals slowly at more distal parts of their 288 dendritic trees (Figure 8A).

289 To address the functional implications of these gradients in the GC and 290 molecular layer, we performed computational modeling of a neuronal network of 291 the cerebellar cortex with integrate-and-fire neurons. The model consisted of one 292 PC and varying number of GCs and MFs (Figure 8A). GCs received randomly 293 determined MF inputs with either tonic (Arenz et al., 2008; van Kan et al., 1993) 294 or bursting (Rancz et al., 2007) in vivo-like spiking sequences. By changing the 295 synaptic weights of the GC to PC synapses, the PC had to acquire a target 296 spiking sequence with regular $80-, 40-$ and $120-\mathrm{Hz}$ firing (Figure 8B). The 297 algorithm for changing the synaptic weights was a combination of a learning 298 algorithm based on climbing-fiber-like punishments and an unbiased 299 minimization algorithm (see Methods).

300 We first compared a model without gradients, where the parameters were set at 301 the average of the experimentally determined values, with a model including all 302 experimentally determined gradients (black and red, respectively, throughout 
303 Figure 8). To measure the difference between the final PC spiking and the target 304 sequence we calculated van Rossum errors using a time constant of $30 \mathrm{~ms}$ 305 (Rossum, 2001) Figure 8C-E). With increasing number of GCs, the final PC 306 spiking sequence resembled the target sequences increasingly better, as 307 illustrated by an average spiking histogram from many repetitions with different 308 random sets of MF inputs for models consisting of 100 and 1000 GCs (Figure 309 8B). As expected, the average minimal van Rossum error (for many repetitions 310 with different random sets of MF inputs) decreased with increasing number of 311 GCs (Figure 8C). For all sizes of the GC population, the average minimal van 312 Rossum error was significantly smaller in the model containing all the 313 experimentally determined gradients compared with the model without any 314 gradients. For example, to obtain the spiking precision of the model containing 315400 GCs with all gradients, the model without gradients required 800 GCs (cf. 316 red arrows in Figure $\mathbf{8 C}$ ). This indicates that for a cerebellum exploiting gradients 317 in the GC layer, the number of GCs can at least be halved to obtain a certain 318 temporal precision compared with a cerebellum containing no gradients.

319 To investigate the relative contribution of each of the gradients, we tested models 320 containing each gradient in isolation, resulting in intermediate van Rossum errors 321 (blue, yellow, and green in Figure 8C,D). The average relative differences 322 between the models across all sizes of the GC populations suggest an almost 323 additive behavior of the individual gradients to the overall performance (Figure $3248 \mathrm{E})$.

325 To further investigate the interplay of the different gradients, we investigated a 326 model containing all gradients, but the connectivity between GCs, PF action 327 potential speed, and PC EPSP kinetics were randomly intermixed (red dashed 328 lines in Figure $8 \mathrm{C}-\mathrm{E}$ ). The network benefits from these intermixed gradients, but 329 maximum optimization can only be obtained with correct connectivity (Figure 330 8E). 
331 The time constant of the van Rossum error can be decreased or increased to 332 investigate spike timing or slower changes in firing rate, respectively. The impact 333 of the gradients increased with increasing time constant (Figure 8-figure

334 supplement $1 A, B)$, indicating that rate coded signaling especially benefits from 335 the here described gradients. To specifically test the effect of gradients on the 336 cerebellum's ability to switch between firing frequencies, we made sigmoid fits 337 around the times of firing rate changes. The transition time ( $t_{T}$; see methods) 338 from these fits showed that models with all gradients showed on average $20 \%$ 339 faster switching between firing frequencies than models without any gradients 340 (Figure 8-figure supplement 1C-F).

341 To further test the influence of gradients on efficient cerebellar processing, we 342 repeated the modeling experiments but used a target sequence with a firing 343 pause (i.e. 80,0 , and $120 \mathrm{~Hz}$ instead of 80,40 , and $120 \mathrm{~Hz}$ ) resulting in similar 344 van Rossum errors and transition time (Figure 8-figure supplement 1G-M). A 345 pause in firing enabled us to quantify the temporal error at the start and the end 346 of the pause (Figure 8-figure supplement $1 \mathbf{N}-\mathbf{Q}$ ), because these spike times 347 have been proposed to be of particular relevance for behavior (Hong et al., 348 2016). Both measures (transition time and temporal error in pause beginning and 349 end) revealed similar results compared with the van Rossum measure, indicating 350 that the speed of 'frequency-switching' and the temporal spiking precision of PCs 351 critically depend on the here described gradients. Thus, our modeling results 352 show that experimentally determined gradients improve the spiking precision, 353 accelerate 'frequency-switching', and increase the storing capacity of the 354 cerebellar cortex.

\section{Discussion}

357 In this study, we describe a gradient in the biophysical properties of superficial to 358 deep GCs, which enables the GC layer to perform a Fourier-like transformation 359 of the MF input. Furthermore, we show that the downstream pathways from GCs 
360 to PCs are specialized for transmitting the frequency band for which the 361 corresponding GCs are tuned to. Finally, computational modeling demonstrates 362 that both the gradients in the GC layer and the specialized downstream pathways 363 improve the spiking precision, accelerate the change of firing frequency of PCs, 364 and increase storing capacity in the cerebellar cortex.

\section{Fourier-like transformation in the cerebellar cortex}

366 Our data demonstrate that outer-zone GCs preferentially fire during MF input with 367 low frequency ('low-frequency' GCs, magenta in Figure 9A), whereas inner-zone 368 GCs preferentially fire during MF input with high frequency ('high-frequency' 369 GCs, green in Figure 9A). The separation of a signal into its frequency 370 components resembles a Fourier transformation (Figure 9B). The analogy with a 371 Fourier transformation has the limitations that (1) a single MF cannot transmit two 372 frequencies simultaneously but only separated in time (cf. example in Figure 9A) 373 and (2) concurrent inputs from two MFs with different frequencies synapsing onto 374 a single GC cannot be separated. Yet, our data indicate that the entire GC layer 375 with several MFs sending various frequencies to numerous GCs can execute a 376 Fourier-like transformation. In analogy to the dispersion of white light in an optical 377 prism into its spectral components, the broadband MF signal is separated into its 378 spectral components with inner- to outer-zone GCs preferentially transmitting the 379 high- to low frequency components, respectively. Such a separation offers the 380 chance to process each frequency component differentially. Indeed, in the 381 molecular layer, the high-frequency components of the MF signal are sent via 382 rapidly conducting axons to proximal parts of the PC dendritic tree. This allows 383 fast (phasic) signals to have a strong and rapid impact on PC firing. On the other 384 hand, low-frequency components of the MF signal are conducted more slowly 385 and elicit slower EPSPs, allowing slow (tonic) signals to have a modulatory 386 impact on PC firing. Our data indicate that, in analogy to the increased storing 387 capacity of digital audio and image compression (Jayant et al., 1993; Wallace, 388 1992), the combination of a Fourier-like transformation in the GC layer and 389 specialized downstream signaling pathways in the molecular layer dramatically 390 reduces the number of required GCs for precise PC spiking (cf. Figure 8). 
391 Furthermore, our data support the 'adaptive filter' theory of the cerebellum, where

392 broadband MF input is differentially filtered by GCs (Dean et al., 2010; Fujita,

393 1982; Singla et al., 2017). Within this framework, our data indicate a gradient in

394 the band-pass filtering properties of GCs. Furthermore, our data could provide an

395 additional explanation for improved motor learning by elevated background

396 activity of MFs (Albergaria et al., 2018), because the elevated MF activity will

397 help overcoming the high threshold of inner-zone GCs, which rapidly and

398 effectively impact PCs via fast conducting PFs at the proximal dendrite.

\section{Axes of frequency specialization in the cerebellum}

400 There are at least two axes of heterogeneity in the cerebellar cortex. First, Zebrin

401 stripes can be observed as parasagittal zones ('medio-lateral' axis) in cerebellar

402 cortex (Apps et al., 2018). Firing rate, firing regularity, synaptic connectivity and

403 even synaptic plasticity seems to differ between PCs in zebrin positive and

404 negative zones (Valera et al., 2016; Wadiche and Jahr, 2005; Xiao et al., 2014;

405 Zhou et al., 2014). Second, there is a lobular organization ('rostro-caudal' axis)

406 as shown here by the functional differences between lobules $\mathrm{V}$ and IX (Figure

407 1-figure supplement 1). GCs in lobule IX are tuned to lower frequencies than 408 GCs in lobule $V$. These findings are largely in line with previous investigations 409 (Heath et al., 2014; Witter and De Zeeuw, 2015a; Zhou et al., 2014), where the 410 anterior cerebellum was identified to process high-frequency or bursting signals, 411 while the vestibulo-cerebellum mainly processed lower frequency or slowly412 modulating inputs. Furthermore, the optimal time intervals for introduction of 413 spike timing dependent plasticity differ between the vermis and the flocculus 414 (Suvrathan et al., 2016).

415 In addition to these two known axes of heterogeneity, we described an axis that 416 is orthogonal to the surface of the cerebellar cortex. This 'depth' axis causes 417 inner-zone GCs to be tuned to higher frequencies than outer-zone GCs. This 418 frequency gradient along the 'depth'-axes is in line with recently described 419 connections of MFs and PC, which specifically target GCs close to the PC layer 420 (Gao et al., 2016; Guo et al., 2016). These connections send slow feedback 
421 signals to the outer-zone GCs, which - according to our framework - are

422 ideally suited to process such slow modulatory signals. Independent of these

423 specialized feedback pathways, MFs exhibit heterogeneity (Chabrol et al., 2015;

424 Bengtsson and Jörntell, 2009). Our data indicate that each type of the

425 heterogeneous MF inputs is split into its frequency components along the depth

426 axis. Our results furthermore predict that superficial GCs, such as the ones

427 imaged recently in the investigation of eye-blink conditioning and reward

428 representation in the cerebellar cortex (Giovannucci et al., 2017; Wagner et al.,

429 2017), would preferentially convey low-frequency signals to PCs and might not

430 be representative for the full range of frequencies present over the depth of the

431 GC layer.

432 Thus, including this new 'depth' axis, there are three orthogonal axes along 433 which the cerebellar cortex is tuned for preferred frequency, indicating the

434 importance of proper frequency tuning of the circuitry.

435 The role of inhibition

436 In the current study we did not investigate molecular layer interneurons, which 437 can have large impact on PC spiking (Blot et al., 2016; Dizon and Khodakhah, 438 2011; Gaffield and Christie, 2017; Mittmann et al., 2005; Sudhakar et al., 2017). 439 However, the spatial arrangement of stellate and basket cell interneurons is 440 consistent with our framework. Although the dendrites of molecular layer 441 interneurons can span the entire molecular layer, the dendrites of basket cells 442 seem to be preferentially located at the inner-zone of the molecular layer 443 (Palkovits et al., 1971; Rakic, 1972), which positions them ideally to receive rapid 444 high-frequency signals of inner-zone PFs. Consistently, they impact PC firing 445 rapidly and efficiently via their pinceaus (Blot and Barbour, 2014). Furthermore, 446 the dendrites of a subset of stellate cells (with their somata located in the outer447 zone molecular layer) are preferentially located at the outer-zone molecular layer 448 (Palkovits et al., 1971; Rakic, 1972), which positions them ideally to receive 449 modulatory low-frequency signals and elicit slow IPSPs in PCs. Furthermore, 450 molecular layer interneurons seem to represent a continuum along the vertical 
451 axis, with a correlation between the vertical location of the soma, axonal boutons,

452 and dendrite location (Sultan and Bower, 1998), which is consistent with the

453 here-described continuum of biophysical properties along the vertical axis of the

454 cortex. Incorporating molecular layer interneurons, their synaptic plasticity and

455 their potential gradients into the frequency-dispersion framework may show a

456 further increase in the dynamic range of frequency separation within the

457 cerebellar cortex over what we have described here (Gao et al., 2012).

\section{Functional implications for the cerebellum}

459 In general, our anatomical and electrophysiological data, combined with our 460 modeling results, show that inner-zone GCs convey high-frequency signals from 461 MFs via rapid pathways to PCs with little filtering. This is in contrast to outer-zone 462 GCs that are tuned to lower frequencies, and which signals undergo more 463 filtering in the PC. These results suggest that the sparse code of GCs (Albus, 464 1971; Billings et al., 2014; Marr, 1969) is in part caused by different frequency 465 tuning of GCs.

466 MF firing frequencies range from <1 to $\sim 1000 \mathrm{~Hz}$ (Arenz et al., 2008; Chadderton 467 et al., 2004; Jörntell and Ekerot, 2006; Rancz et al., 2007; van Kan et al., 1993). 468 Many previous modeling studies investigating cerebellar function considered the 469 activity of each MF as a constant digital value (Albus, 1971; Babadi and 470 Sompolinsky, 2014; Brunel et al., 2004; Clopath et al., 2012; Marr, 1969), a 471 constant analog value (Chabrol et al., 2015; Clopath and Brunel, 2013), or spike 472 sequences with constant frequency (Billings et al., 2014; Cayco-Gajic et al., 473 2017; Steuber et al., 2007). We focused on the time-varying aspects of MF 474 integration in GCs, and therefore implemented a model with a corresponding 475 large range of MF input frequencies that could change over time. It would be 476 interesting to elucidate in, as much previous models, consisting of more uniform 477 MF inputs, would benefit from the here-observed biophysical gradients.

478 To implement these gradients in a model we used a simplified cerebellar circuitry 479 that does not consider active dendrites (Llinás and Sugimori, 1980) or the tonic 
480 activity of PCs (Raman and Bean, 1997). It will therefore be interesting to 481 investigate if the here-observed gradients in the GC and molecular layer improve 482 the performance of more complex models of the cerebellar cortex (De Schutter 483 and Bower, 1994a; Garrido et al., 2013; Masoli et al., 2015; Medina et al., 2000; 484 Rossert et al., 2015; Spanne and Jörntell, 2013; Steuber et al., 2007; Sudhakar 485 et al., 2017; Walter and Khodakhah, 2009; Yamazaki and Tanaka, 2007). 486 Furthermore, it remains to be investigated whether gradients in the GC layer also 487 improve models that aim to explain tasks such as eye-blink conditioning (Mauk 488 and Buonomano, 2004) and vestibulo-ocular reflexes (du Lac et al., 1995)

489 Our model simulated the learning that PCs undergo to acquire specific firing 490 frequencies in response to GC input. PC firing rate and spiking precision have 491 been shown to be closely related to movement (Brown and Raman, 2018; 492 Sarnaik and Raman, 2018). Our results show that the same temporal spiking 493 precision or the same frequency switching speed can be obtained with 494 approximately half the number of GCs when GC gradients are implemented 495 (Figure 8). Taking into account the large number of cerebellar GCs in the brain 496 (Herculano-Houzel, 2009; Williams and Herrup, 1988), a significant reduction in 497 the number of GCs could represent an evolutionary advantage to minimize 498 neuronal maintenance energy (Howarth et al., 2012; Isler and van Schaik, 2006). 499 Therefore, the dramatic increase in storing capacity for precise PC spiking 500 provides an evolutionary explanation for the emergence of gradients in the 501 neuronal properties.

\section{Functional implications for other neural networks}

503 Based on the described advantages of the Fourier transformation for rapid and 504 storing-efficient information processing, we hypothesize that other neural 505 networks also perform Fourier-like transformations and use segregated 506 frequency-specific signaling pathways. To our knowledge this has rarely been 507 shown explicitly, but similar mechanisms might operate, for example, in the 508 spinal cord network: descending motor commands from the pyramidal tract send 509 broadband signals to motoneurons with different input resistances resulting from 
510 differences in size. This enables small motoneurons to fire during low-frequency

511 inputs and large motoneurons only during high-frequency inputs (Henneman et

512 al., 1965). Furthermore, specialized efferent down-stream signaling pathways

513 innervate specific types of muscles with specialized short-term plasticity of the

514 corresponding neuromuscular junctions (Wang and Brehm, 2017).

515 In the hippocampus, frequency preferences of hippocampal neurons are well 516 established enabling segregation of compound oscillatory input into distinct

517 frequency components (Pike et al., 2000). Furthermore, there is increasing 518 evidence that previously considered homogeneous population of neurons exhibit 519 gradients in the neuronal properties (Cembrowski and Spruston, 2019), such as 520 the intrinsic electrical properties and synaptic connectivity in CA3 pyramidal 521 neurons (Galliano et al., 2013). The heterogeneity furthermore enables functional 522 segregation of information streams for example in CA1 pyramidal neurons 523 (Soltesz and Losonczy, 2018). Finally, in the neocortex, gradients in anatomical 524 and biophysical properties were recently uncovered (Fletcher and Williams, 525 2019).

526 In summary, our findings contribute to the growing body of evidence that the 527 neurons of a cell layer can exhibit systematic functional heterogeneities with 528 differential tuning of neurons along gradients. Our data furthermore suggest that 529 such gradients facilitate complex transformation of information, such as Fourier530 like transformations, to cope with a broad temporal diversity of signals in the 531 central nervous system.

533 Material and Methods

534 Electrophysiology

535 Parasagittal 300- $\mu \mathrm{m}$ thick cerebellar slices were prepared from P21-P30 (young 536 animals) or from P80-P100 (old animals) C57BL/6 mice of either sex as 537 described previously (Ritzau-Jost et al., 2014);(Delvendahl et al., 2015). Animals 538 were treated in accordance with the German and French Protection of Animals 
539 Act and with the guidelines for the welfare of experimental animals issued by the

540 European Communities Council Directive. The extracellular solution for the

541 whole-cell measurements contained (in $\mathrm{mM}$ ): $\mathrm{NaCl} 125, \mathrm{NaHCO}_{3} 25$, glucose 20 ,

$542 \mathrm{KCl} 2.5, \mathrm{CaCl}_{2} 2, \mathrm{NaH}_{2} \mathrm{PO}_{4} 1.25, \mathrm{MgCl}_{2} 1$ (310 mOsm, pH 7.3 when bubbled with

543 Carbogen $\left.\left(95 \% \mathrm{O}_{2} / 5 \% \mathrm{CO}_{2}\right)\right)$. For outside-out measurements of potassium

544 currents (Figure 2), $150 \mu \mathrm{M} \mathrm{CdCl}_{2}$ and $1 \mu \mathrm{M}$ TTX were added to the external

545 solution to block voltage-gated calcium channels and sodium channels,

546 respectively. The intracellular solution contained in $\mathrm{mM}$ : K-Gluconate $150, \mathrm{NaCl}$

547 10, K-Hepes 10, Mg-ATP 3, Na-GTP 0.3, EGTA 0.05 (305 mOsm, pH 7.3). A

548 liquid junction potential of $+13 \mathrm{mV}$ was corrected for. All electrophysiological

549 measurements were performed with a HEKA EPC10 amplifier (HEKA Elektronik,

550 Lambrecht/Pfalz, Germany) under control of the Patchmaster software. All

551 measurements were performed at $34-37^{\circ} \mathrm{C}$.

552

553 Current clamp recordings in GCs

554 Action potentials were evoked in current-clamp mode by current pulses

555 (amplitude 20-400 pA, duration $300 \mathrm{~ms}$ ). To determine the input resistance, 556 subthreshold current pulses were applied from -20 to +20 pA in 2 pA steps. The

557 resistance of the solution-filled patch-pipettes was between 6-12 $\mathrm{M} \Omega$ and the

558 mean series resistance was not compensated during the measurement. Data 559 were sampled at $200 \mathrm{kHz}$.

560

561 Outside-out recordings in GCs

562 To reliably clamp potassium currents from the soma of GCs (Figure 2), 563 potassium currents were measured in outside-out patches pulled from the soma 564 of inner and outer GCs by applying $10 \mathrm{~ms}$ voltage steps from -90 to $+60 \mathrm{mV}$ with $56510 \mathrm{mV}$ increments at an intersweep interval of $1 \mathrm{~s}$. The intersweep holding 566 potential was $-90 \mathrm{mV}$. Data were sampled at $100 \mathrm{kHz}$.

568 Compound action potentials in PFs

569 For the detection of compound action potentials in PFs, two pipettes (tip 
570 resistances 1-4 M 2 ) filled with extracellular solution and connected to the patch-

571 clamp amplifier were positioned within the molecular layer of horizontally cut

572 slices of the cerebellar vermis. The average distance between two recording

573 electrodes was $143 \pm 5 \mu \mathrm{m}$. Compound action potentials were evoked by voltage

574 stimulation $(100 \mathrm{~V})$ for $100 \mu \mathrm{s}$ with a third pipette connected to an accumulator

575 powered stimulation device (ISO-Pulser ISOP1, AD-Elektronik, Buchenbach,

576 Germany). 40 to 80 stimulations delivered at $1 \mathrm{~Hz}$ were averaged and analyzed.

577

578 Excitatory postsynaptic potentials in PC

579 Excitatory postsynaptic potentials (EPSPs) in PC were elicited by voltage 580 stimulation of the PFs within the inner, middle or outer third of the molecular layer 581 from horizontally cut cerebellar slices (Figure 7). $10 \mu \mathrm{M}$ SR95531 was added to 582 the external solution to block $\mathrm{GABA}_{\mathrm{A}}$ receptors. The stimulation pipette was filled 583 with extracellular solution, and the voltage was adjusted between 6 to $25 \mathrm{~V}$ to 584 elicit EPSPs with an amplitude between 1 and $2 \mathrm{mV}$. EPSPs were measured 585 after a single $100 \mu \mathrm{s}$ voltage stimulation or 5 stimulations (100 $\mu$ s duration) at a 586 frequency of 100 and $500 \mathrm{~Hz}$. Averages of 30 trains per stimulation protocol were 587 used for data analysis.

588

589 Excitatory postsynaptic currents in GCs

590 To measure evoked EPSCs from GCs (Figure 3-figure supplement 1), 90-100

591 days-old mice were used. GCs from inner- or outer-zone from lobule $V$ were held 592 at resting conditions and MF axons were stimulated at $1 \mathrm{~Hz}$ with a second 593 pipette. The average stimulation voltage was $36 \pm 3 \vee$ for outer-zone GCs and 37 $594 \pm 3$ V for inner-zone GCs.

595

\section{Dynamic Clamp of MF conductance in GCs}

597 In order to analyze the response of GCs on in-vivo like MF inputs, we used a 598 dynamic clamp implemented with the microcontroller Teensy 3.6 599 (https://www.pjrc.com) as described by Desai et al. (Desai et al., 2017). The 600 Teensy was programmed using the Arduino integrated development environment 
601 with the code provided by Desai et al (2017) and modified for our need as

602 described in the following.

603

604 The time course of MF conductance was

605

$$
G_{E P S C}(t)=G_{\text {max }} A_{\text {norm }}\left(-e^{-\frac{t}{\tau_{r}}}+\sum_{i=1}^{3} a_{i} e^{-\frac{t}{\tau_{i}}}\right)
$$

606 where the exponential rise time $\left(\tau_{r}\right)$ was $0.1 \mathrm{~ms}$, the decay time constants $\left(\tau_{1}, \tau_{2}\right.$,

607 and $\tau_{3}$ ) were $0.3,8$, and $40 \mathrm{~ms}$, respectively, and the relative amplitude of the

608 decay components $\left(a_{1}, a_{2}\right.$, and $\left.a_{3}\right)$ were $0.7,0.26$, and 0.04 , respectively. The

609 peak conductance $\left(G_{\max }\right)$ was $1 \mathrm{nS}$ (Hallermann et al., 2010) and the

610 normalization factor $\left(A_{\text {norm }}\right)$ was 0.518 , which was numerically calculated to

611 obtain a peak amplitude of 1 . The kinetics of the MF conductance were chosen to

612 reproduce the measured mixed AMPA and NMDA EPSC kinetics of single

613 EPSCs (Figure 3-figure supplement 1) and trains of EPSCs (Baade et al.,

614 2016). The short-term plasticity during Poisson sequence of spikes was

615 implemented by changing $G_{\max }$ according to a simple phenomenological model

616 (Tsodyks and Markram, 1997) assuming a release probability p po of 0.4 (Ritzau-

617 Jost et al., 2014). Facilitation was implemented as an increase in the release

618 probability according to $\mathrm{p}_{\mathrm{r}}=\mathrm{p}_{\mathrm{r}}+0.2^{*}\left(1-\mathrm{p}_{\mathrm{r}}\right)$ and decaying back to $\mathrm{p}_{\mathrm{ro}}$ with a time

619 constant of $12 \mathrm{~ms}$ (Saviane and Silver, 2006). Depression was implemented

620 according to a recovery process with a time constant of 25 ms, which

621 approximates a biexponential recovery process of $12 \mathrm{~ms}$ and $2 \mathrm{~s}$ (Hallermann et

622 al., 2010; Saviane and Silver, 2006). The resulting short-term plasticity

623 reproduced previously obtained data with regular spiking ranging from 20 to 1000

$624 \mathrm{~Hz}$ (Baade et al., 2016; Hallermann et al., 2010; Ritzau-Jost et al., 2014).

625

626 The microcontroller was programmed to implement the MF conductance and its

627 short-term plasticity with Poisson distributed spike times with a constant

628 frequency ranging from 30 to $500 \mathrm{~Hz}$ for $300 \mathrm{~ms}$ (Figure 3). In each cell, each

629 frequency was applied five times. 
631 To investigate the response to sinusoidally varying input frequencies (Figure 4), 632 the target frequency of the Poisson process $(F)$ was varied on a logarithmic scale 633 according to:

$$
F(t)=\exp \left(\log \left(F_{\text {min }}\right)+\left(\log \left(F_{\text {max }}\right)-\log \left(F_{\text {min }}\right)\right)(0.5+0.5 \sin (2 \pi t / T))\right)
$$

635 where the minimal and maximal frequency $\left(F_{\min }\right.$ and $\left.F_{\max }\right)$ were 30 and $300 \mathrm{~Hz}$,

636 respectively, and the duration of the sine wave cycle $(T)$ was $1 \mathrm{~s}$. In each cell, 10

637 cycles were applied consecutively. The histogram of the spike times (Figure 4B)

638 was averaged across the last six cycles of all cells. The vector strength and 639 phase angle (Kan et al., 1993) were calculated as the absolute value and the

640 argument of the complex number $\rho(i=\sqrt{-1})$ :

$$
\rho=\frac{1}{N} \sum_{n=1}^{N} e^{i 2 \pi \frac{t_{n}}{T}}
$$

642 where $t_{n}$ are the spike times of all $N$ spikes per experiment and $T$ the cycle

643 duration (1 s).

644

645 Electron Microscopy

646 Four C57BL/6 mice of either sex with an age between P23-P28 were sacrificed,

647 followed by transcardial perfusion with saline and consecutively a fixative 648 containing $4 \%$ paraformaldehyde and $2 \%$ glutaraldehyde in phosphate-buffered 649 saline (PBS). After removal of the brain, the tissue was allowed to post-fix over 650 night at $4^{\circ} \mathrm{C}$ and sagittal sections of the cerebellum were prepared at a thickness 651 of $60 \mu \mathrm{m}$ using a Leica microtome (Leica Microsystems, Wetzlar, Germany). The 652 sections were stained in $0.5 \%$ osmium tetroxide in PBS for 30 min followed by 653 dehydration in graded alcohol and another staining step with $1 \%$ uranyl acetate 654 in 70\% ethanol. After further dehydration, the tissue was embedded in durcupan 655 (Sigma-Aldrich), which was allowed to polymerize for $48 \mathrm{~h}$ at $56^{\circ} \mathrm{C}$ between 656 coated microscope slides and cover glasses. Regions of interest were identified 657 by light microscopy, cut and transferred onto blocks of durcupan to obtain ultra- 
658 thin sections using an Ultramicrotome (Leica Microsystems). Ultra-thin sections 659 were transferred onto formvar-coated copper grids and stained with lead citrate.

660 Ultrastructural analysis was performed using a Zeiss SIGMA electron microscope 661 (Zeiss NTS, Oberkochen, Germany) equipped with a STEM detector and ATLAS 662 software.

663

664 Measurement of parallel-fiber axon diameter

665 Electron micrographs were manually analyzed in a blind manner (numbered by 666 masked randomization) and each micrograph was divided into eight identically 667 sized fields. The diameter of each parallel-fiber axon was measured as the 668 longest chord in one or two of these fields. Cross sections with visible active 669 zones or mitochondria were excluded from analysis.

\section{Dil Injections and GC tracking}

672 Six P20 CD1 mice were anesthetized with isoflurane (4\%). An incision of the skin 673 to expose the skull and a hole was manually drilled using a 25G needle above 674 the desired injection site. Injections of small amounts of Dil (1,1-dioctadecyl$6753,3,3,3$ tetramethylindocarbocyanine perchlorate, ThermoFisher Scientific, 10\% 676 in N,N-dimethylformamide) were performed using a broken glass pipette 677 connected to a picospritzer II (Parker Instrumentation). $24 \mathrm{~h}$ after injection, 678 animals were sacrificed and transcardially perfused with $4 \%$ paraformaldehyde in 679 PBS. The cerebellum was dissected, fixed overnight, and embedded in $4 \%$ 680 agarose in PBS. 150- $\mu \mathrm{m}$ thick sections were then cut in the transverse or sagittal 681 plane using a vibratome (VT1000, Leica microsystems). Z-Stacks (1 $\mu \mathrm{m}$ steps) 682 were acquired using a confocal microscope (Leica SP5 II, 63x objective). GCs 683 were traced from their soma to the axonal bifurcation of PFs. (Average stack 684 depth: $84 \pm 20 \mu \mathrm{m})$. GC axons were reconstructed using the 'Simple Neurite 685 Tracer' plugin (Longair et al., 2011) in Fiji (ImageJ, NIH, USA). This plugin 686 allowed us to assess the continuity of axons between several cross-sections. GC 687 ascending axons were then fully traced and measured within the Z-limits of 688 image sections. The size of the different layers of cerebellar cortex was 
689 reconstructed in each Z-stack. To avoid variability, all distances were normalized

690 to the corresponding molecular layer height.

691

692 Data analysis

693 Data were analyzed using custom-written procedures in lgor Pro software

694 (WaveMetrics, Oregon, USA). Intrinsic properties of GCs were determined from

695 the injected currents that elicited the largest number of action potentials. The

696 action potential threshold was defined as the membrane voltage at which the first

697 derivative exceeded $100 \mathrm{~V} \mathrm{~s}^{-1}$, the minimal action potential peak was set as -20

$698 \mathrm{mV}$ and the minimal amplitude to $20 \mathrm{mV}$. All action potentials with a half-width

699 smaller than $50 \mu$ s and larger than $500 \mu$ s were excluded. Action potential

700 frequency and half-width were calculated from the first three action potentials.

701 Membrane capacitance, resting membrane potential and series resistance were

702 read from the amplifier software (HEKA) after achieving the whole-cell

703 configuration. Input resistance $\left(R_{\text {in }}\right)$ was analyzed from alternating subthreshold

704 current injections from -20 to 20 pA (2 pA steps). The voltage was plotted against

705 injected current and a spline interpolation was performed to obtain the slope at

706 the holding membrane potential (0 pA current injection).

707 Peak-current from outside-out patches was determined from voltage steps (-90

708 to $+60 \mathrm{mV}$ ) with Fitmaster software (HEKA). Steady-state inactivation was

709 determined from the last $2 \mathrm{~ms}$ of the respective sweep. Cells were only included

710 if $50 \mathrm{pA}<\mathrm{I}_{\max }<1 \mathrm{nA}$ to exclude potential whole-cell measurements and

711 membrane-vesicles.

713 EPSP measurements from PCs and EPSC measurements from GCs were 714 analyzed with the Fitmaster software (HEKA). For PC EPSPs, $20-80 \%$ rise time 715 and time to peak were determined from the average of 30 individual single 716 EPSPs. GCs EPSCs were averaged from 25 traces. To obtain the decay 717 kinetics, single EPSPs/EPSCs were fitted with either one or two exponentials. 718 The weighted time constant was calculated as: 
720 Paired-pulse ratio was determined between the first and the $5^{\text {th }}$ EPSP after

721 stimulation with $100 \mathrm{~Hz}$ trains. Single EPSCs from inner- and outer-zone GCs

722 were averaged and fitted with two exponentials. The decay kinetics and

723 amplitude of the grand-average was used to implement the MF EPSCs for the

724 Dynamic Clamp.

725

\section{Neuronal networking modeling}

727 The neuronal network consisted of varying numbers of MF inputs, GCs and one 728 PC and was implemented in Matlab (The MathWorks, Inc, Natick, 729 Massachusetts, R2017a). For each simulation, a random set of MF inputs was 730 generated. This input was then fed to a layer of integrate-and-fire GCs. A 731 modeled PC then used the output of these GCs for trial-to-trial learning. The PF-

732 to-PC synaptic weights were optimized with the aim to make the PC spiking 733 sequence similar to the target sequence. In the following, each component of the 734 model is explained in detail. MF inputs

737 To simulate in vivo like MF firing patterns, half of the MFs fired tonically (van Kan 738 et al., 1993) and the other half fired bursts (Rancz et al., 2007). All MF spike 739 trains were modeled first by generating a 'threshold trace'. For tonically firing 740 MFs, this threshold trace was a Gaussian function with a peak and standard 741 deviation chosen from uniform distributions ranging between 10 and $100 \mathrm{~Hz}$ and 7420.2 and $0.5 \mathrm{~s}$ respectively, and a peak time point between 0 and $0.5 \mathrm{~s}$. For burst 743 firing MFs, the threshold trace was an exponential function with a peak randomly 744 chosen between 600 and $1200 \mathrm{~Hz}$, a decay time constant of $30 \mathrm{~ms}$ and a peak 745 between 0 and $0.5 \mathrm{~s}$. The threshold trace was then evaluated against random 746 numbers from a uniform distribution to determine the occurrence of a spike. To 747 accelerate the simulations the sampling time interval was $1 \mathrm{~ms}$. 
749 GC properties

750 GCs were implemented as integrate and fire models with the following 751 parameters: the membrane resistance was linearly varied between $450 \mathrm{M} \Omega$ for 752 inner GCs to $800 \mathrm{M} \Omega$ for outer GCs (Figure $1 \mathrm{H}$ ) and the threshold was linearly 753 varied between $-37 \mathrm{mV}$ for inner GCs to $-42 \mathrm{mV}$ for outer GCs (Figure 1G). For 754 the models without the GC gradient, these values were set to the mean of the 755 values for the inner and outer GC (i.e. $625 \mathrm{M} \Omega$ and $-39 \mathrm{mV}$ ). The reset potential 756 was set to $-90 \mathrm{mV}$ and the membrane potential to $-80 \mathrm{mV}$.

PF properties

759 To simulate a different action potential propagation speed, the GC spike times 760 were delayed by a value linearly varied between 0 for inner and $3 \mathrm{~ms}$ for outer 761 GCs. The delay was calculated as the difference in conduction time required to 762 travel $5 \mathrm{~mm}$ with a speed of 0.28 and $0.33 \mathrm{~m} \mathrm{~s}^{-1}$ (Figure $4 \mathrm{~F}$ ). Even with this 763 anatomically rather too large PF length (Harvey and Napper, 1991), the PF 764 propagation speed has only a small impact on the model performance (see e.g. 765 blue lines and bars in Figure $7 \mathrm{C}-\mathrm{E}$ ), arguing against a big impact of PF 766 conduction delays (Braitenberg et al., 1997) at least in our model approach.

Synaptic connections and properties

769 Each MF was connected to 10 GCs and each GC received 2 MF inputs, i.e. the 770 number of MF was $1 / 5$ of the number of GCs. Since our model consists only of 771 'active' MFs, we chose only 2 and not 4 MFs per GCs (Billings et al., 2014). The 772 MF to GC synapse was implemented as a model with one pool of vesicles with a 773 release probability of 0.5 and a vesicle recruitment time constant of $13 \mathrm{~ms}$ 774 (Hallermann et al., 2010). Synaptic facilitation was implemented by increasing 775 the release probability after each spike by 0.2 decaying to the resting release 776 probability with a time constant of $12 \mathrm{~ms}$ (Saviane and Silver, 2006). The 777 synaptic conductance had exponential rise and decay time constants of 0.1 and $7782 \mathrm{~ms}$, respectively, and a peak amplitude of $1.9 \mathrm{nS}$ (Silver et al., 1992). 779 Correspondingly, the GC to PC synapse was implemented as a model with one 
780 pool of vesicles with a release probability $\left(\mathrm{p}_{\mathrm{ro}}\right)$ of 0.4 and a vesicle recruitment 781 time constant of $50 \mathrm{~ms}$. Synaptic facilitation was implemented by increasing the 782 release probability after each spike by 0.2 decaying to the resting release 783 probability with a time constant of $50 \mathrm{~ms}$ (Doussau et al., 2017; Isope and 784 Barbour, 2002; Valera et al., 2012). The synaptic conductance had exponential 785 rise time constant between 0.5 and $2 \mathrm{~ms}$ and decay time constant between 17.5 786 and $70 \mathrm{~ms}$ for inner- and outer-zone GCs, respectively (Figure 6). The peak 787 amplitude was adjusted to equalize the charge of the EPSC and to generate an 788 approximately correct number of PC spikes (with the initial start values, i.e. all 789 GC to PC synaptic weight factors = 1) by linearly varying between 0.5 and 0.15 790 nS for inner- and outer-zone GCs, respectively.

791

792 PC properties

793 The PC was implemented as an integrate and fire model with a membrane 794 resistance of $15 \mathrm{M} \Omega$, resting membrane potential of $-50 \mathrm{mV}$, and a firing 795 threshold of $-45 \mathrm{mV}$. Spontaneous firing of PCs (Raman and Bean, 1997) was

796 not implemented, and the only inputs to drive PCs to threshold were the GC-to797 PC EPSCs.

799 Target sequence and van Rossum measure

800 Based on in vivo firing patterns (Witter and De Zeeuw, 2015a), an arbitrary target 801 firing sequence of 80,40 , and $120 \mathrm{~Hz}$ for 300,100 , and $100 \mathrm{~ms}$, respectively, 802 was chosen. The distance between the PC and the target spiking sequence was 803 quantified with the van Rossum error (van Rossum, 2001). Both spiking 804 sequences were convolved with an exponential kernel with a decay time constant 805 of $30 \mathrm{~ms}$ (or values ranging from 2 to $300 \mathrm{~ms}$ in (Figure 8-figure supplement 806 1A,B). The van Rossum error was defined as the integral of the square of the 807 difference between these two convolved traces. We also tested another 808 algorithm to calculate the van Rossum error (Houghton and Kreuz, 2012), which $809 \mathrm{C}++$ code was taken from http://pymuvr.readthedocs.io/ and incorporated into 810 Matlab via the MEX function and results were comparable. 
812 Learning and minimization algorithm

813 For each random set of MF inputs, the GC to PC synaptic weights were changed

814 according to the following algorithm with the aim to minimize the van Rossum

815 error between the PC spiking sequence and the target sequence. The initial

816 values of the synaptic weights were 1, and values were allowed to change

817 between 0 and 100. First, an algorithm was used that was based on supervised

818 learning (Raymond and Medina, 2018) to punish the GCs that have spikes that

819 precede unwanted PC spikes. Subsequently, an unbiased optimization of the GC

820 to PC synaptic weight was performed using the patternsearch() algorithm of

821 Matlab to minimize the van Rossum error. To increase the chance that a global

822 (and not local) minimum was found, the minimization of the routine was repeated

823 several times with random starting values. Other optimization routines such as a

824 simplex [(fminsearch() of Matlab) or a genetic algorithm (ga() of Matlab)] revealed

825 similar results. To exclude the possibility that the differences in the minimal van

826 Rossum error between models with and without gradients were due to a bias in

827 our learning algorithm, we performed a set of simulations with networks

828 consisting of less than 100 GCs, in which we skipped the learning algorithm and

829 only used unbiased minimization algorithm. This resulted in similar difference in

830 the minimal van Rossum error between models with and without gradients,

831 indicating that the learning algorithm was not biased towards one type of model.

832 For networks with consisting of more than $100 \mathrm{GCs}$ the pre-learning was required

833 to facilitate the finding of the global minimum.

835 Analysis of modeling results

836300 different sets of random MF inputs were used to determine 300 statistically

837 independent minimal van Rossum values for each of the models with different

838 number of GCs and different number of implemented gradients (illustrated as

839 mean \pm SEM in Figure 7C). Comparing different models with the same set of MF

840 input (using the nonparametric paired Wilcoxon signed-rank statistical test) the

841 difference was significant $(p<0.001)$ for all of the models and all number of GCs. 
842 The van Rossum errors were then normalized to the mean of the error of the

843 model without gradients (Figure 7D). The values in Figure 8D were fitted with

844 cubic spline interpolation using the logarithm of the number of GCs as abscissa.

846 To quantify the transition time between two target frequencies of the $\mathrm{PC}$, the

847 spike histogram was fitted with the equation

$$
f(t)=80+\frac{-80+40}{1+e^{-(t-200) / t_{T}}}+\frac{-40+120}{1+e^{-(t-300) / t_{T}}}
$$

849 where $f$ is the spike frequency in $\mathrm{Hz}$ and $t$ the time in ms. The transition time $t_{T}$

850 corresponds to the $23 \%$ to $77 \%$ decay and rise time for the transition from 80 to

$85140 \mathrm{~Hz}$ and from 40 to $120 \mathrm{~Hz}$, respectively.

852

853 Sensitivity of model parameters

854 We verified that our conclusions do not critically depend on specific parameters

855 of the model. For example, decreasing the simulation time interval from $1 \mathrm{~ms}$ to $856100 \mu \mathrm{s}$, resulted in difference of the best van Rossum error of $21 \%$ between 857 models with and without gradients consisting of $100 \mathrm{GC}$, compared with a 858 difference of $17 \%$ between the corresponding models with the default simulation 859 time interval of $1 \mathrm{~ms}$ (cf. Figure 8D). With 4 MFs per GCs (not 2) the difference 860 of the best van Rossum error was $15 \%$ between models with and without 861 gradients consisting of 100 GC (17\% with 2 MF per GC). With a membrane 862 resistance of the $P C$ of $100 \mathrm{M} \Omega$ (not $15 \mathrm{M} \Omega$ ) the difference of the best van 863 Rossum error was $23 \%$ between models with and without gradients consisting of $864100 \mathrm{GC}(17 \%$ with $15 \mathrm{M} \Omega)$. Finally, changing the target sequence to 80,0 , and $865120 \mathrm{~Hz}$ (not 80, 40, and $120 \mathrm{~Hz}$ ) resulted in very similar results as obtained with 866 the original target (compare Figure Figure 8C-E with Figure 8-figure 867 supplement $1 \mathrm{H}-\mathrm{J}$ and Figure 8-figure supplement 1 D-F with Figure 8868 figure supplement $1 \mathrm{~K}-\mathrm{M})$. Statistical testing

871 All data are expressed as mean \pm SEM. The number of analyzed cells is 872 indicated in the figures. To test for statistically significant differences of normally 
873 distributed data, we performed ANOVA or Student's t-tests and provided the $\mathrm{P}$

874 value ( $\mathrm{P}_{\mathrm{ANOVA}}$, and $\mathrm{P}_{\mathrm{T} \text {-TEST, }}$, respectively) above the bar-graphs. To test the 875 differences between single groups we performed a Tukey post-hoc test and 876 provided the corresponding $P$ value in the figure legend $\left(P_{\text {Tukey }}\right)$. For not normally 877 distributed data we performed a Mann-Whitney test. 


\section{Figures and figure legends:}
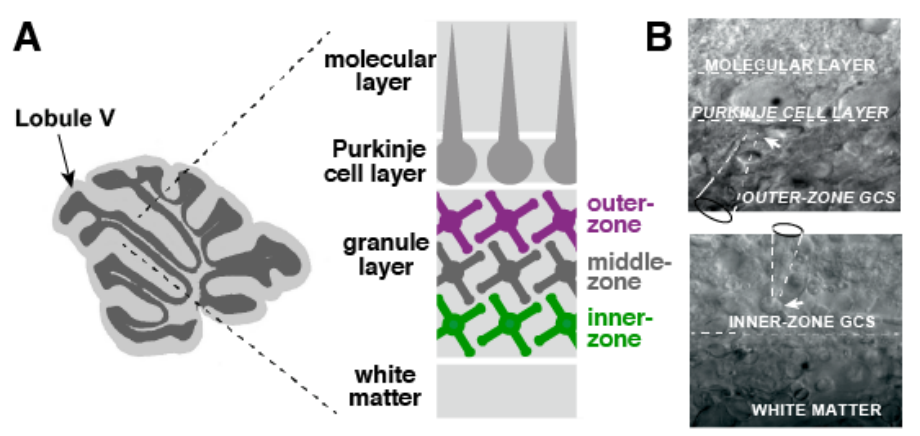

C
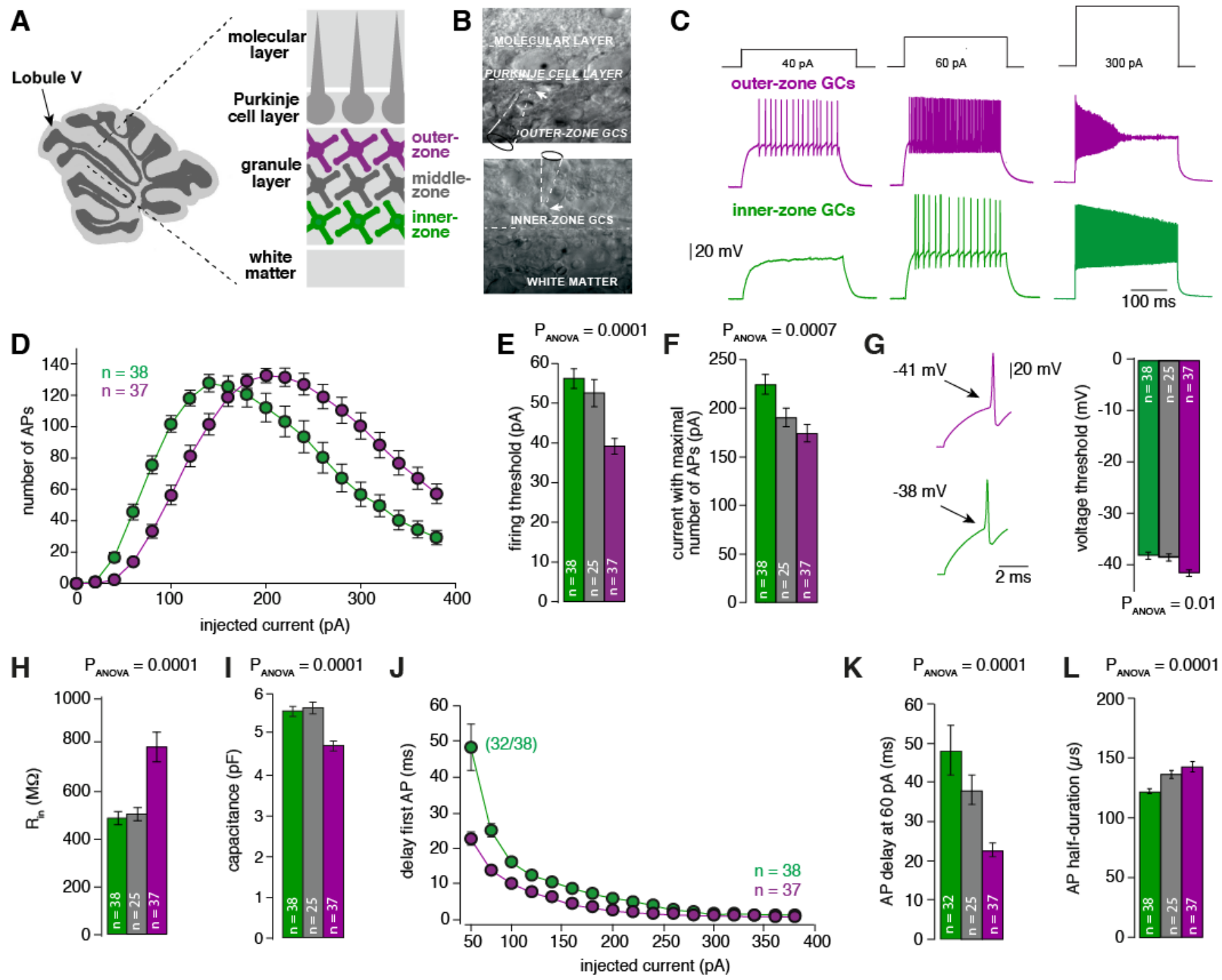

881 Figure 1: A gradient in the biophysical properties of inner- to outer-zone

\section{GCs}

A. Scheme of a parasagittal slice from the cerebellar cortex were lobule $V$ is indicated by an arrow. Enlargement shows a schematic representation of the white matter, the GC, PC and molecular layer of the cerebellar cortex. Throughout the manuscript, inner-zone GCs (close to the white matter) are depicted in green, the middle-zone GCs in grey, and the outer-zone GC (close to the PCs) in magenta. acute cerebellar slices during recordings from outer- (top) and an inner-zone GCs (bottom). The pipette is indicated with dashed line. 
892 C. Example current-clamp recordings from an outer-zone GC (magenta, top) and an inner-zone GC (green, bottom) after injection of increasing currents (40 pA, $60 \mathrm{pA}$ and $300 \mathrm{pA})$.

D. Number of action potentials from inner- (green, $n=38$ ) and outer(magenta, $\mathrm{n}=37$ ) zone GCs plotted against the injected current. Note that the maximum number of action potentials is similar but outer-zone GCs achieved the maximum firing rate with a lower current injection.

E. Average current threshold for action potential firing of inner- (green), middle- (grey) and outer-zone GCs (right) $\left(P_{\text {Tukey }}=0.0001\right.$ for inner- vs outer-zone GCs).

902

903

F. Average current needed to elicit the most number of action potentials

904 for of inner- (green), middle- (grey) and outer-zone GCs (magenta)

905 $\left(P_{\text {Tukey }}=0.0005\right.$ for inner- vs outer-zone GCs).

906

907

908

909

$\mathrm{H}$. Average input resistance of inner- (green), middle- (grey) and outer-

G. Left: example action potentials from an inner- and outer-zone GC with the indicated (arrows) mean voltage-threshold for firing action potentials. Right: Comparison of the average voltage threshold for action potential firing ( $P_{\text {Tukey }}=0.002$ for inner- vs outer-zone GCs). zone GCs ( $P_{\text {Tukey }}=0.0001$ for inner- vs outer-zone GCs).

I. Average capacitance of inner-, middle- and outer-zone GCs $\left(\mathrm{P}_{\text {Tukey }}=\right.$ 0.0001 for inner- vs outer-zone GCs).

J. Delay time of the first action potential plotted against the injected current. Note, only 32 of 38 inner-zone GCs were firing action potential at a current injection of $60 \mathrm{pA}$.

K. Delay of the first action potential of inner- (green), middle- (grey) and outer-zone GCs at a current injection of $60 \mathrm{pA}$ ( $P_{\text {Tukey }}=0.0001$ for inner- vs outer-zone GCs).

L. Average action potential half-duration of inner- (green), middle- (grey) and outer-zone GCs ( $P_{\text {Tukey }}=0.0001$ for inner- vs outer-zone GCs). 

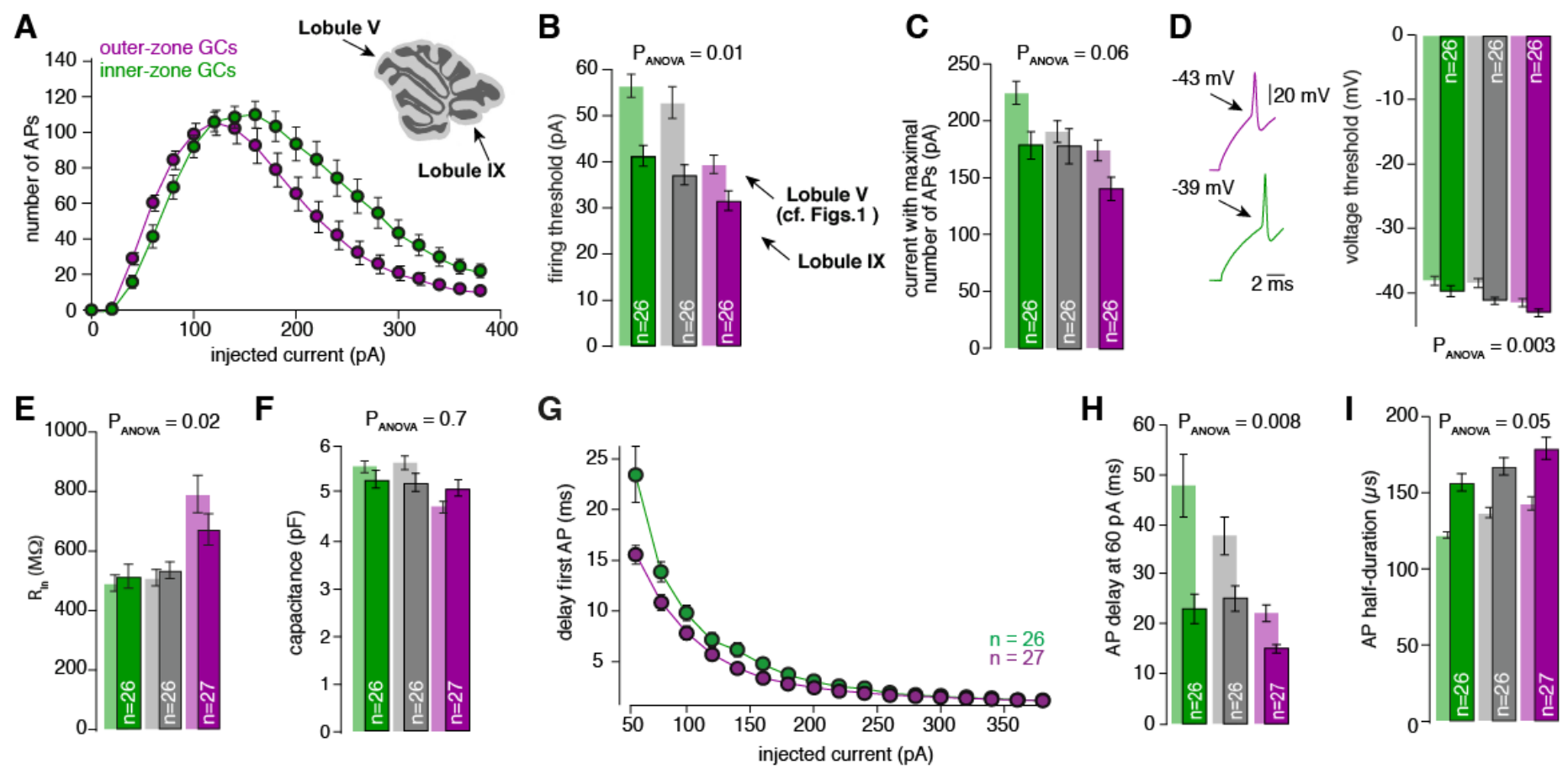

923 Figure 1-figure supplement 1: The gradient in the biophysical properties 924 of GCs and PFs is preserved throughout the cerebellar cortex.

925 A. Number of action potentials (APs) from inner (green) and outer (magenta) zone GCs plotted against the injected current. The maximum number of action potentials is similar but with more current

929 injection, inner-zone GCs fire more action potentials. Inset: An image of a cerebellar slice shows were the lobule $\mathrm{V}$ and lobule IX are

931 indicated by arrows.

932

B. Bar graphs represent the firing threshold of GCs from inner (dark-

933 green), middle (dark-grey) and outer-zone (dark-magenta). The lightcolored bar graphs in the background are the data from lobule $\mathrm{V}$ shown in Figure 1. Firing threshold is higher in inner- compared to outer-zone GCs from lobule IX, and with the same current injection, GCs from lobule IX fire action potentials faster compared to lobule $V$. The numbers of recorded GCs for lobule IX $(n)$ are indicated $\left(P_{\text {Tukey }}=\right.$ 0.007 for inner- vs outer-zone GCs).

940

C. Average current needed to elicit the maximum number of action

941 potentials for of inner- (green), middle- (grey) and outer-zone GCs

942 (magenta) $\left(P_{\text {Tukey }}=0.002\right.$ for inner- vs outer-zone GCs).

943

D. Left: example action potentials from an inner- and outer-zone GC with the indicated (arrows) mean voltage threshold for firing action 
potentials. Right: Voltage threshold to elicit action potentials in inner-, middle- and outer-zone GCs from lobule IX compared with data from lobule $\mathrm{V}$. Voltage threshold for outer-zone GCs is lower compared to inner-zone GCs from lobule IX ( $P_{\text {Tukey }}=0.002$ for inner- vs outer-zone GCs).

949 E. Input resistance of GCs from outer-zone of lobule IV is higher compared to inner- and middle zone GCs. But there is no difference between the input resistance of GCs from lobule $\mathrm{V}$ and IX $\left(\mathrm{P}_{\text {Tukey }}=\right.$ 0.02 for inner- vs outer-zone GCs).

F. Average capacitance of inner-, middle- and outer-zone GCs. In contrary to lobule $V$ there is no difference in the capacitance of GC from inner-, middle, or outer-zone $\left(P_{\text {Tukey }}=0.7\right.$ for inner- vs outer-zone GCs).

G. Delay of the first action potential plotted against the injected current. inner-, middle- and outer-zone GCs from lobule IX compared to lobule $\mathrm{V}\left(\mathrm{P}_{\text {Tukey }}=0.05\right.$ for inner- vs outer-zone GCs).

I. The action potential half-duration of inner-zone (dark-green) GCs from lobule IX is shorter compared to middle (dark-grey)- and outer-zone (dark-magenta) GCs. Compared to lobule $V$ (faded-green, faded grey and faded magenta), the GCs from lobule IX showed a broader action potential half-width ( $P_{\text {Tukey }}=0.09$ for inner- vs outer-zone $\left.G C s\right)$. 
A

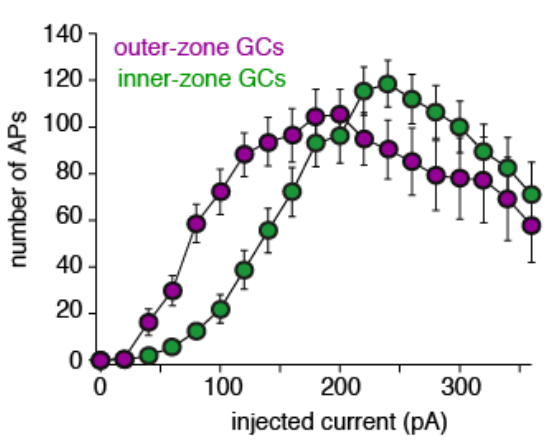

$\mathbf{E}$
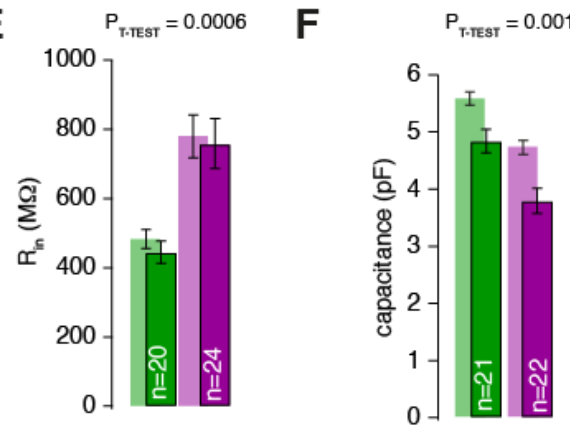

B

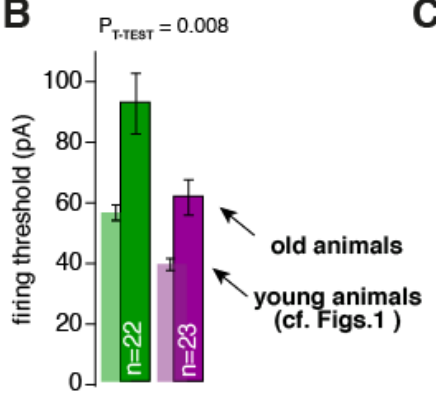

F $\quad P_{\text {T.TEST }}=0.001$

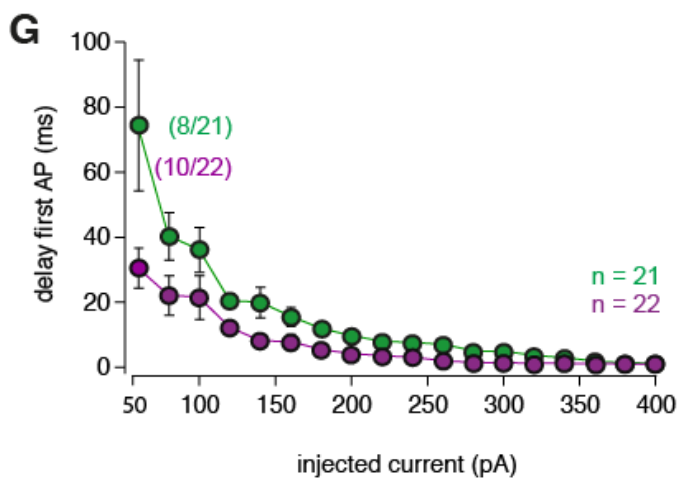

D
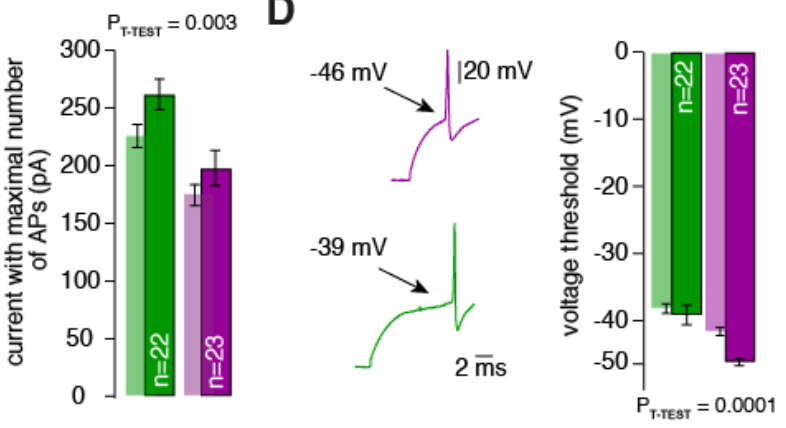

H
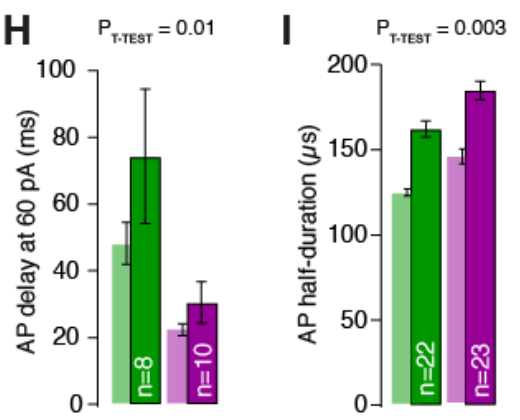

968 Figure 1-figure supplement 2: The gradient in the biophysical properties 969 of GCs and PFs is also found in 3-month-old animals.

970 A. Number of action potentials (APs) from inner (green) and outer (magenta) zone GCs plotted against the injected current. The maximum number of action potentials is similar, but with more current

973 injection, inner-zone GCs fire more action potentials. For all the measurements GCs from lobule $V$ were used.

975

B. Bar graphs represent the firing threshold of GCs from inner (dark-

976 green), and outer-zone (dark-magenta). The light-colored bar graphs in the background are the data from lobule $V$ in young (21-30 daysold) animals shown in Figure 1. Firing threshold is higher in inner compared to outer-zone GCs from old animals.

980

C. Average current needed to elicit the maximum number of action

981 potentials for of inner- (green), and outer-zone GCs (magenta).

982

Left: example action potentials from an inner- and outer-zone GC with

983 the indicated (arrows) mean voltage-threshold for firing action potentials. Right: voltage threshold to elicit action potentials in inner-, middle- and outer-zone GCs from old animals compared with data from young animals.

987

E. Input resistance of GCs from outer-zone is higher compared to inner-

988 zone GCs. But there is no difference between the input resistance of GCs from young and old animals. 
$990 \quad$ F. Average capacitance of inner- and outer-zone GCs. In agreement with the data from the young animals, inner-zone GCs have a higher

992 capacitance compered to outer-zone GCs.

993

994 Delay of the first action potential plotted against the injected current. Since the mean current threshold is higher compared to young animals only 8 out of 21 GCs from inner- and 10 out of 22 GCs from outer-zone already fired action potentials at a current injection

$\mathrm{H}$. Delay of the first action potential after a current injection of $60 \mathrm{pA}$ from inner-, middle- and outer-zone GCs from lobule IX compared to lobule $\mathrm{V}$. old animals is shorter compared with outer-zone (dark-magenta) GCs. 
A
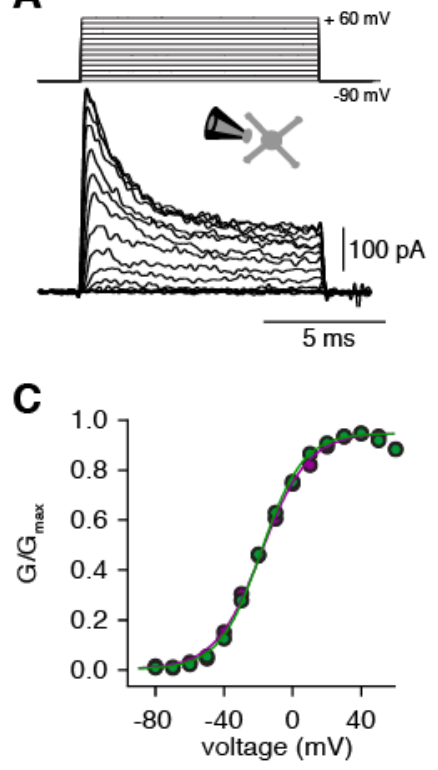

B

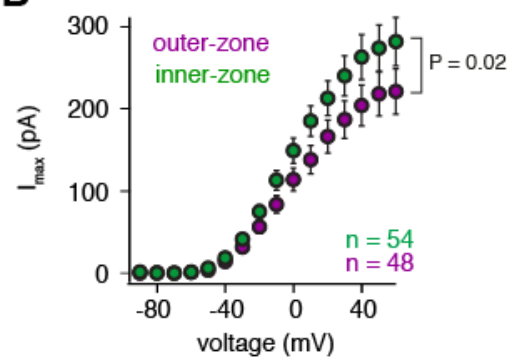

D

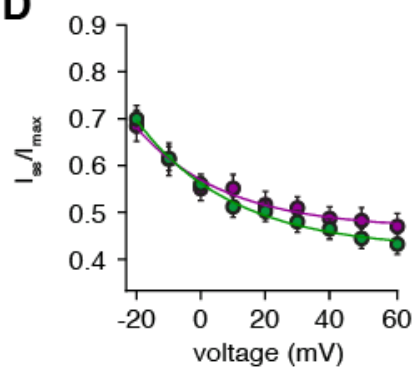

1004

1005

1006

1007

1008

1009

1010

1011

1012

1013

1014

1015

1016

1017

1018

Figure 2: Voltage-gated potassium currents are larger at inner-zone GCs

A. Example potassium currents from outside-out patches of cerebellar GCs evoked by voltage steps from -90 to $+60 \mathrm{mV}$ in $10 \mathrm{mV}$ increments with a duration of $10 \mathrm{~ms}$. All recordings were made in the presence of $1 \mu \mathrm{M}$ TTX and $150 \mu \mathrm{M} \mathrm{CdCl} 2$ to block voltage-gated sodium and calcium channels, respectively.

B. Average peak potassium current $\left(I_{\max }\right)$ plotted versus step potential of inner (green) and outer-zone (magenta) GCs. Significance level was tested with a Mann-Whitney Test and the $P$ value is indicated in the figure. Note, that the $P$ value shows the significance level at $60 \mathrm{mV}$.

C. Average normalized peak potassium conductance $\left(G / G_{\max }\right)$ versus step potential of inner (green) and outer-zone (magenta) GCs.

D. Average steady-state current ( $\mathrm{I}_{\mathrm{ss}}$, mean current of the last $2 \mathrm{~ms}$ of the $10 \mathrm{~ms}$ depolarization) normalized to the peak current $\left(I_{\max }\right)$ versus step potential of inner (green) and outer-zone (magenta) GCs. 


\section{A}
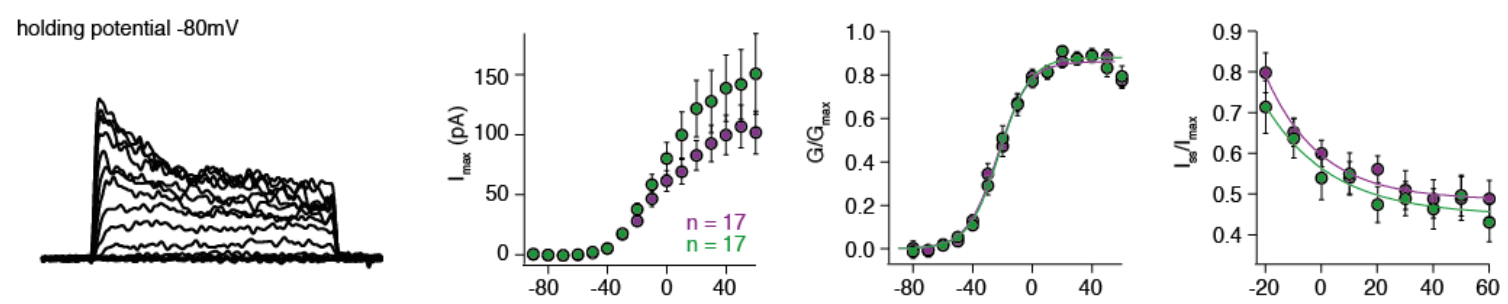

B

holding potential $-70 \mathrm{mV}$
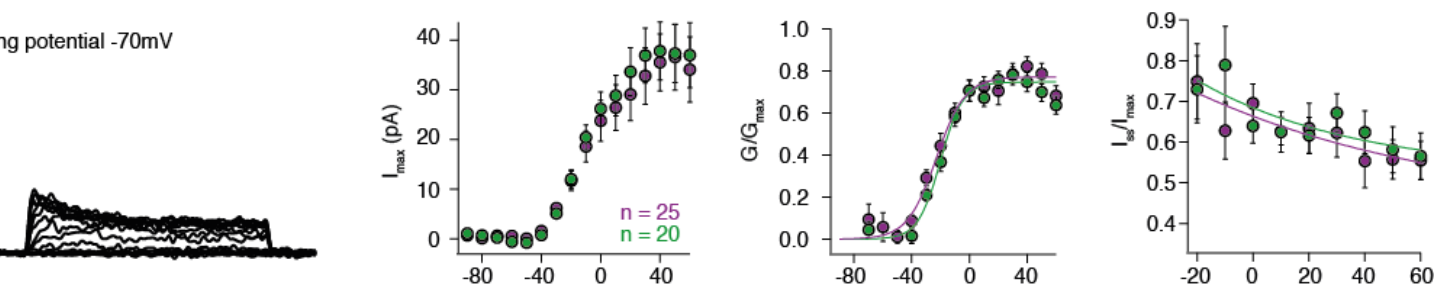

\section{C}

holding potential $-60 \mathrm{mV}$
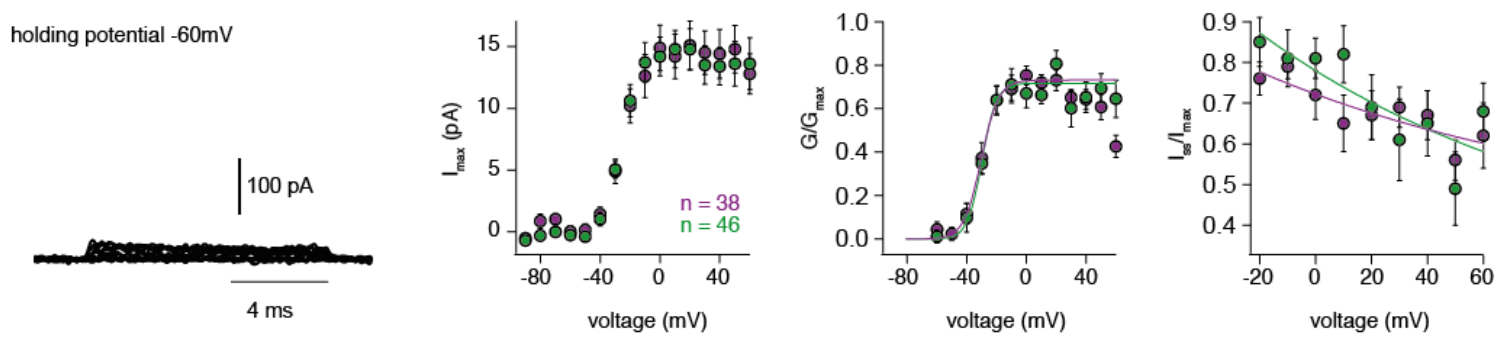

1021 Figure 2-figure supplement 1: Steady-state activation and inactivation are similar for inner and outer GCs.

1023

1024

A - C left: Example potassium currents from outside-out patches of cerebellar

1025 GCs evoked by voltage steps from -90 to $+60 \mathrm{mV}$ in $10 \mathrm{mV}$ increments with a duration of $10 \mathrm{~ms}$. The intersweep holding potential varied between $-80 \mathrm{mV}(\mathbf{A})$, $70 \mathrm{mV}$

(B) and $-60 \mathrm{mV}$

(C). Panels

1028 relationship (first pane) the normalized conductance (second panel) and the normalized inactivation behavior (third panel). The number of measured cells are indicated in the figure. 
A

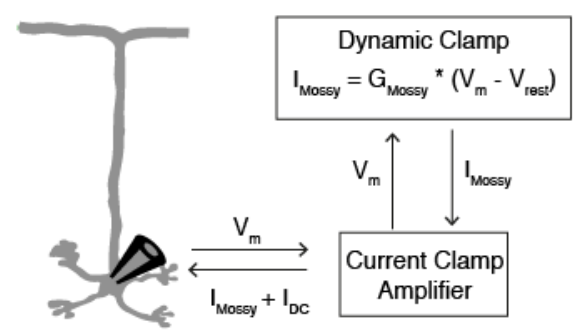

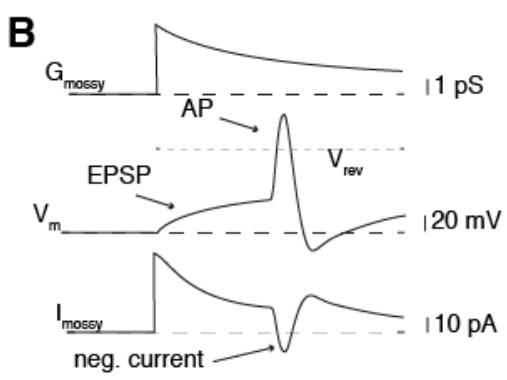

C

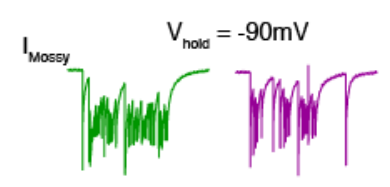

$\stackrel{ }{1}$

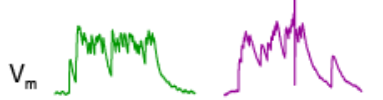

inner-zone GCs outer-zone GCs

D

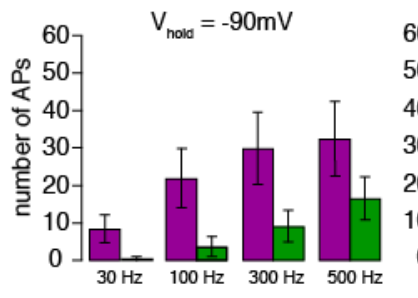

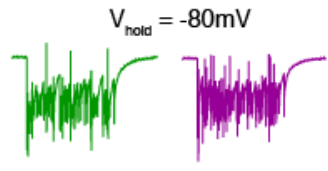
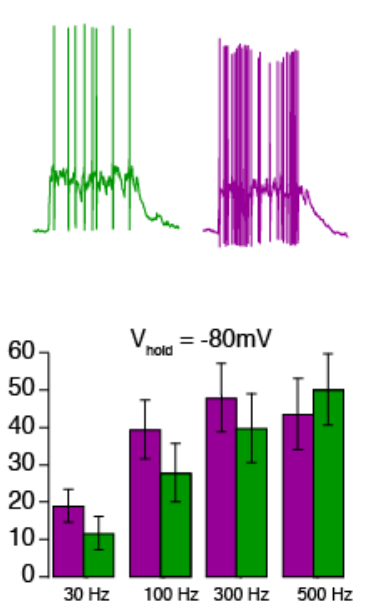
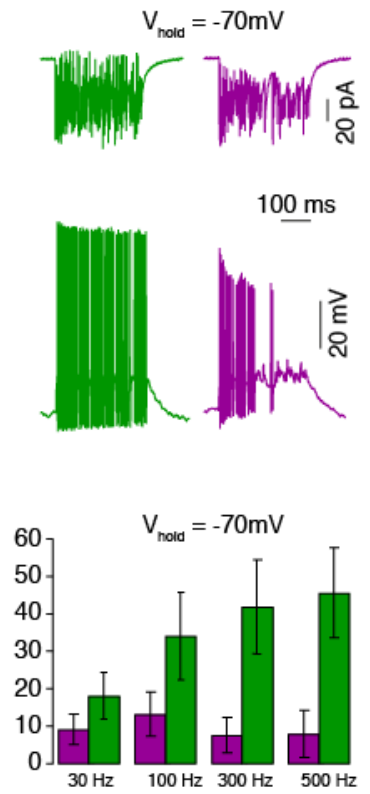

1033

1034

1035

1036

1037

1038

1039

1040

1041

1042

1043

1044

1045

1046

1047

1048

Figure 3: MF inputs are differentially processed by inner and outer GCs

A. Schematic representation of the Dynamic Clamp system.

B. Illustration of MF conductance $\left(G_{\text {mossy }}\right), G C$ membrane potential $\left(V_{m}\right)$, and MF current ( $\left.I_{\text {mossy }}\right)$ for the dynamic clamp technique. Note the prediction of a negative current during the action potential as apparent in the experimental traces in panel $\mathrm{C}$.

C. Example dynamic clamp recordings of inner- (green) and outer-zone (magenta) GCs at different holding potentials (-90 mV left; $-80 \mathrm{mV}$ middle and $-70 \mathrm{mV}$ right) at a stimulation frequency of $100 \mathrm{~Hz}$. Upper trace represents the poisson-distributed MF-like EPSCs. Lower trace shows the measured EPSPs and action potentials.

D. Average number of measured action potentials (APs) after mossy-fiber like EPSC injection at different frequencies and the indicated holding potentials. 


\section{0}

A

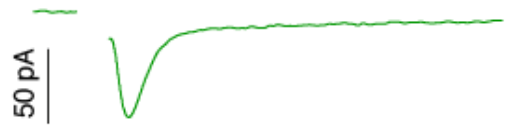

m
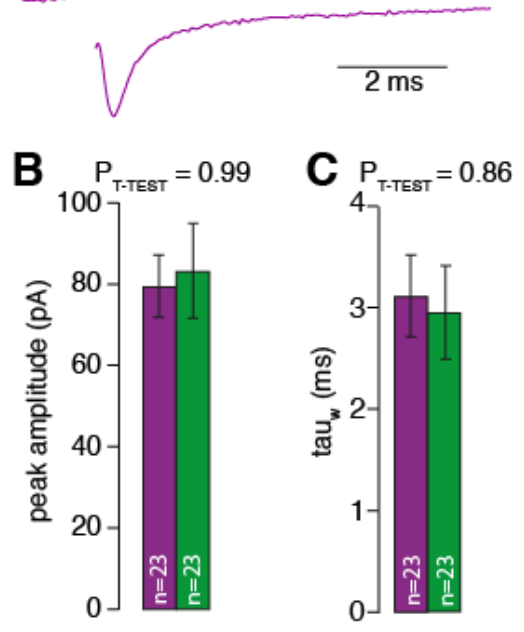

Figure 3-figure supplement 1: MF input is similar for inner- and outerzone GCs.

A. Examples of single EPSCs measured from inner- (green) and outerzone GCs (magenta) after $1 \mathrm{~Hz}$ stimulation of MF axons. Stimulation artifacts were removed.

B. Average amplitude of EPSCs from inner- (green) and outer-zone GCs.

C. Weighted decay time (see material and methods) of EPSCs from inner- (green) and outer-zone (magenta). 


\section{1}

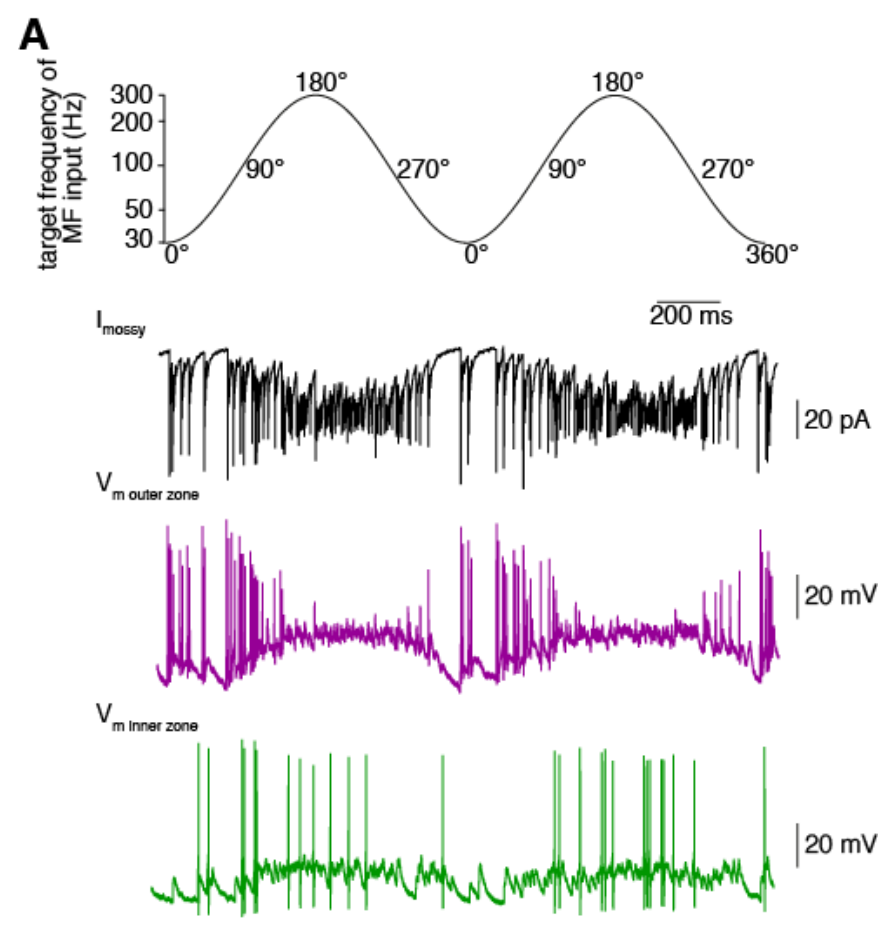

B

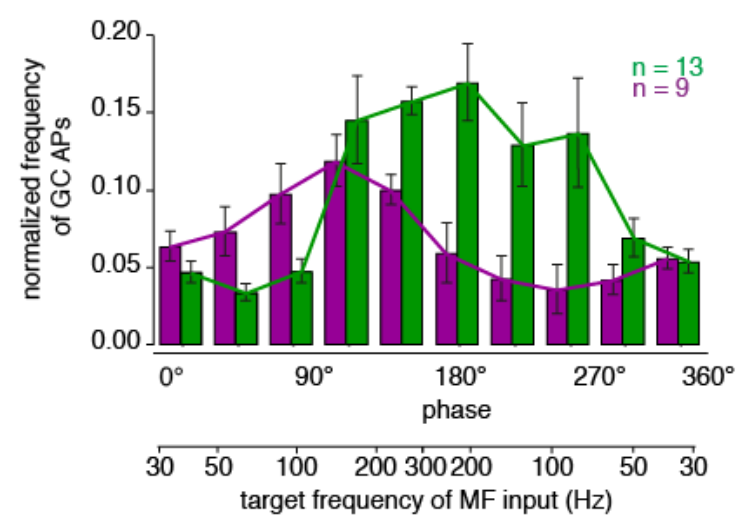

1061 Figure 4: Fourier-like transformation of MF input frequency

1062

1063

1064

1065

1066

1067

1068

1069

1070

1071

1072
A Target frequency of the dynamic clamp MF-like sinusoidal EPSC input current. The frequencies ranged from 30 to $300 \mathrm{~Hz}$ and the duration was $1 \mathrm{~s}$. The degree values denote the phase angle. Black: example trace of poisson-distributed MF-like input current. Magenta and green: Dynamic clamp measurement of outer- and inner-zone GCs, respectively, after stimulation with a MF-like current shown in black at a holding potential of approximately $-70 \mathrm{mV}$.

B Histogram of the normalized frequency of action potentials (APS) fired by GCs at different time intervals during 1s stimulation with poissondistributed MF-like currents shown in A. Each bar represents a time window of 100 ms. Green: inner-zone GCs, magenta: outer-zone GCs. 

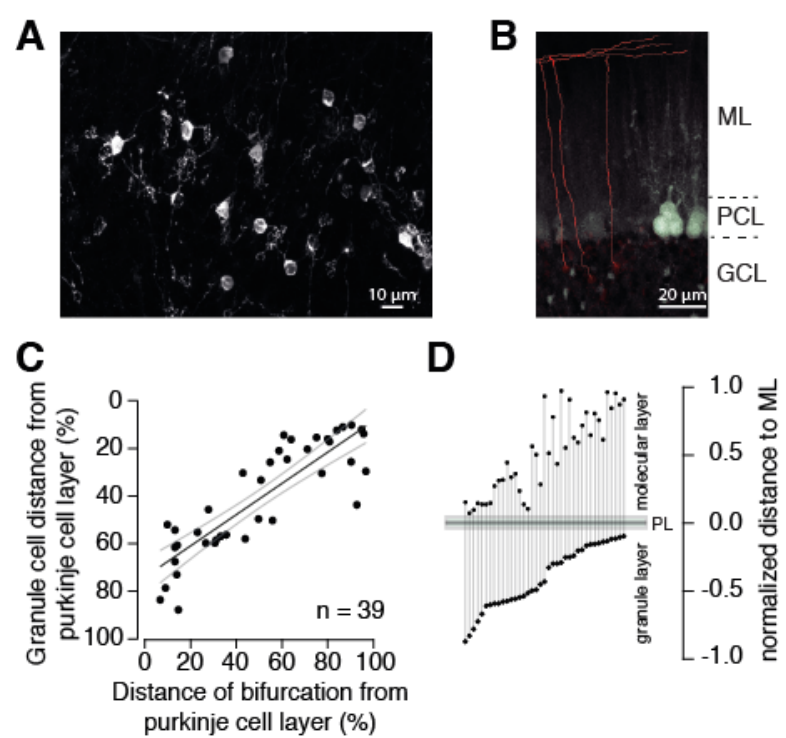

Figure 5: The position of PFs is correlated with the position of GC somata

A. Example of GCs labeled with Dil $24 \mathrm{~h}$ post injection. Numerous GCs from inner-, middle-, and outer-zone were labeled.

1078

B. Example of traced axons from different GCs from the outer zone. The axon was traced (red) from the cell soma to the bifurcation side in the molecular layer. Stained cell bodies of PFs are also visible (white). ML: molecular layer; PCL: Purkinje cell layer; GCL: granule cell layer.

C. The distance between labeled GCs and the PC layer strongly correlated with the distance between the axon bifurcation and the PC layer (Pearson's correlation coefficient $r=-0.862 ; p<0.001$ ). Solid black line depicts the linear interpolation and the grey lines represent SEM of the fit. The number of recorded GCs ( $n$ ) is indicated.

D. Position of the GC somata from the granular layer of each traced cell linked to the position of the bifurcation side in the molecular layer. To avoid variability, all the distances were normalized to the corresponding molecular layer hight. 
A

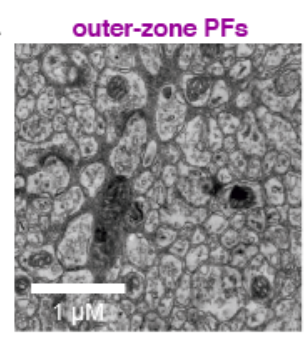

D

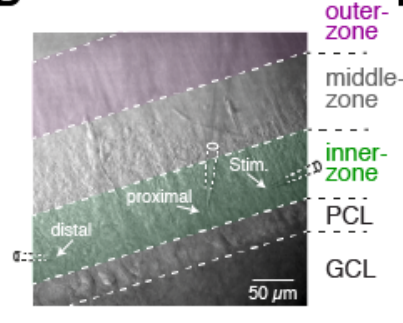

B

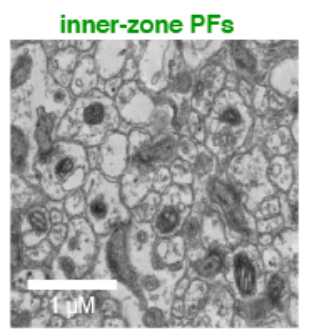

E

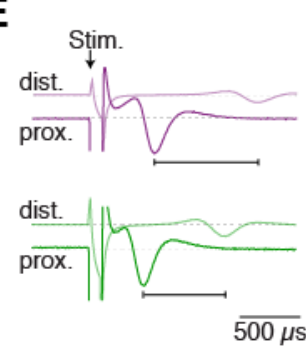

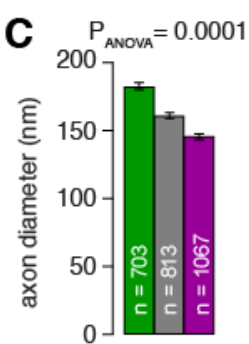

F $\quad P_{\text {ANova }}=0.0001$

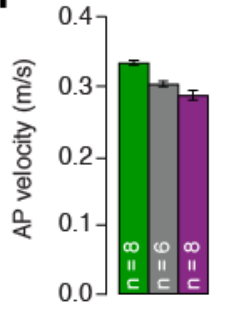

Figure 6: Inner-zone PFs have larger diameter and higher action potential

A. Electron microscopic image of the outer (A) and inner zone (B) of sagittal sections through the molecular layer.

C. Summary of axon diameters in the inner (green), middle (grey), and outer zone (magenta) of the molecular layer $\left(P_{\text {Tukey }}=0.0001\right.$ for inner- vs outerzone GCs).

D. DIC image of the molecular layer superimposed with a schematic illustration of the experimental setup to measure compound action potentials from PFs. Compound action potentials were evoked by a stimulus electrode (left) and recorded by a proximal and distal recording electrode (middle, right).

E. Example traces used to determine the conduction velocity between inner-, middle-, and outer-zone PFs. The time difference between the compound action potential arriving at the proximal electrode (solid traces) and the distal electrode (faint traces) was used to determine the velocity. The time was shorter for inner-zone PFs (green) compared with outer-zone PFs (magenta).

F. Summary of action potential (AP) velocity in inner-, middle- and outerzones $\left(P_{\text {Tukey }}=0.0001\right.$ for inner- vs outer-zone GCs). 
A

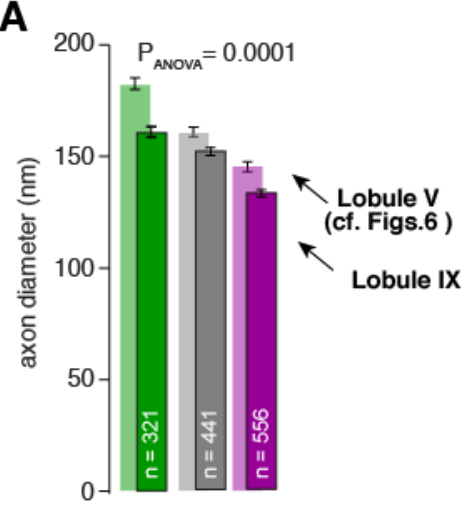

B

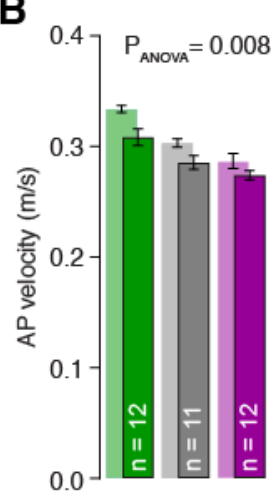

Figure 6-figure supplement 1: Differences in axon diameter and action 1118 potential velocity are also found in lobule IX.

1119

1123

1124

1125

1126

1127
A. Summary of axon diameters in the inner (green), middle (grey), and outer zone (magenta) of the molecular layer $\left(P_{\text {Tukey }}=0.0001\right.$ for innervs outer-zone GCs). The light-colored bar graphs in the background are the data from lobule $\mathrm{V}$ shown in Figure 6.

B. Summary of action potential (AP) velocity in inner-, middle- and outerzones of PF from molecular layer of lobule IX $\left(\mathrm{P}_{\text {Tukey }}=0.0001 \mathrm{for}\right.$ inner- vs outer-zone GCs). 

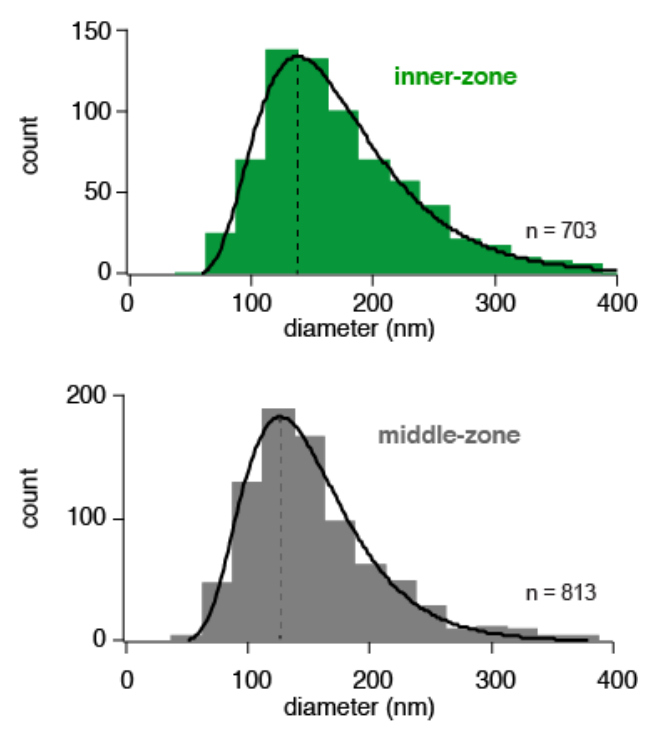

\section{Figure 6-figure supplement 2: Histogram of the axon diameters.}

1131 Histograms of the diameter of inner-, middle- and outer-zone axons in the 1132 molecular layer, indicating that the change in axon diameter is not due to an 1133 increased fraction of larger diameter axons from non-GC cells (e.g. Lugaro cells). 1134 Instead the entire distribution of the axon diameters is shifted between zones.

1135 Data were fit with a skewed Gaussian function: $a e^{\left[-\log (2)\left(\left(\log \left(1+2 b \frac{d-d 0}{d s}\right) / b\right)^{2}\right)\right]}$, 1136 where $a$ is the amplitude, $d$ the diameter, and $d 0$ the diameter at the peak. $d s$ 1137 and $b$ represent parameters related to the width and the skewness, respectively. 1138 The peak is indicated by a vertical line.

1139

1140 

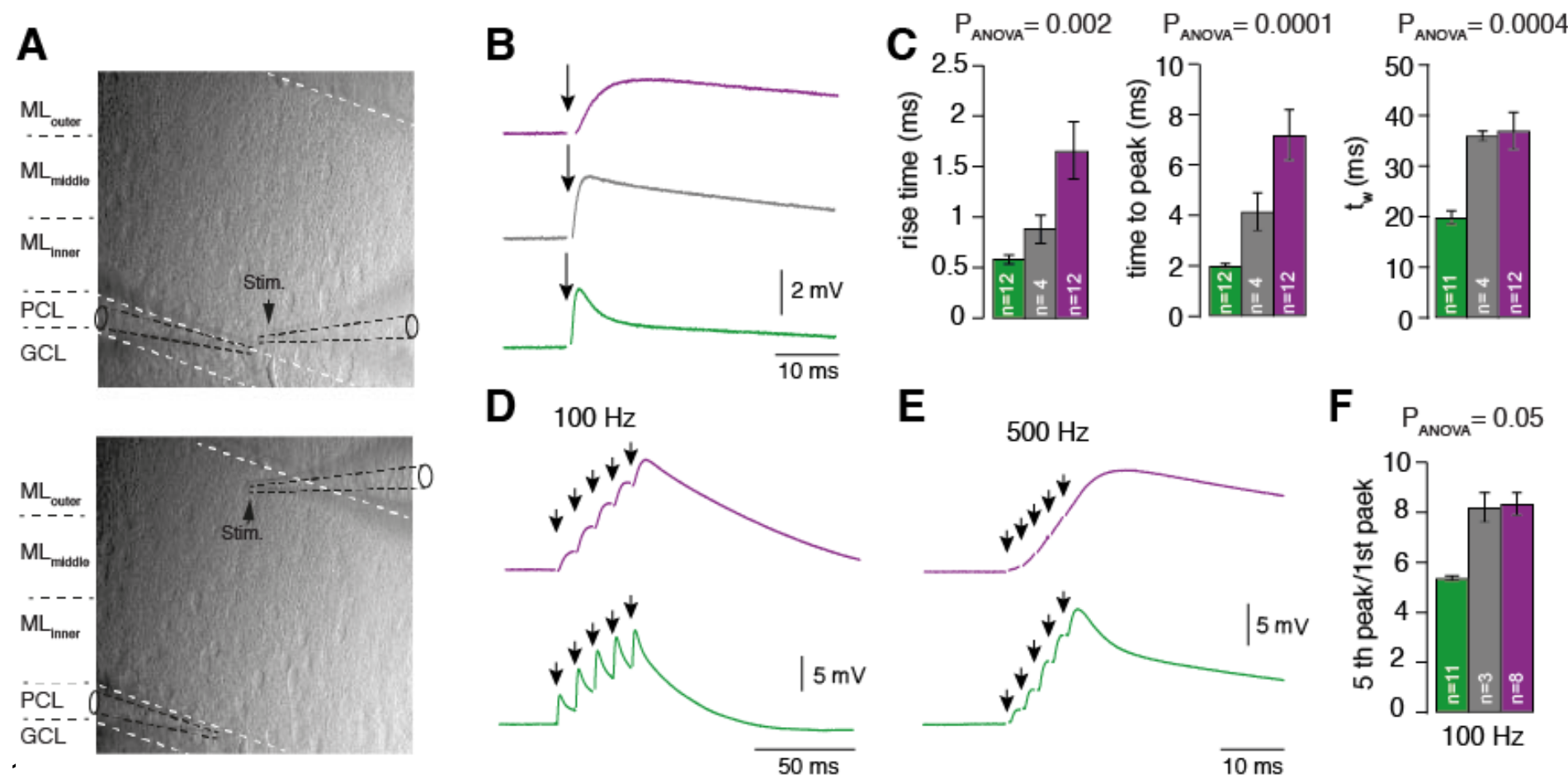

1142 Figure 7: PCs process inner-, middle-, and outer-zone PF inputs 1143 differentially

1144

1145

1146

1147

1148

1149

1150

1151

1152

1153

1154

1155

1156

1157

1158

1159

1160
A. DIC image of the molecular layer superimposed with a schematic illustration of PC recordings while stimulating inner (top) and outerzone PFs (bottom). Shown are the GC layer (GL), PC layer (PC) and molecular layer $(\mathrm{ML})$.

B. EPSPs measured at the PC soma after stimulation $(1 \mathrm{~Hz})$ of inner(green), middle- (grey), and outer-zone PFs (magenta).

C. Average $20 \%$ to $80 \%$ rise time, time to peak and weighted timeconstant of PC EPSPs after stimulation of inner- (green; $n=12$ ), middle- (grey; $n=4$ ) and outer-zone PFs (magenta; $n=12$ ) as shown in $B\left(P_{\text {Tukey }}=0.001 ; P_{\text {Tukey }}=0.0001 ; P_{\text {Tukey }}=0.0004\right.$ for inner- vs outerzone GCs, respectively).

D-E. Example traces of EPSPs from a PC after five impulses to inner(green) and outer-zone PFs (magenta) at $100 \mathrm{~Hz}$ (D) and $500 \mathrm{~Hz}(\mathrm{E})$.

F. Average paired-pulse ratio measured in PCs after five $100-\mathrm{Hz}$ stimuli at inner- (green; $n=11$ ), middle- (grey, $n=3$ ) and outer- zone PFs (magenta, $n=8)\left(P_{\text {Tukey }}=0.054\right.$ for inner- vs outer-zone GCs). 
bioRxiv preprint doi: https://doi.org/10.1101/764886; this version posted September 10, 2019. The copyright holder for this preprint (which was not certified by peer review) is the author/funder, who has granted bioRxiv a license to display the preprint in perpetuity. It is made available under aCC-BY 4.0 International license.

\section{7}

A
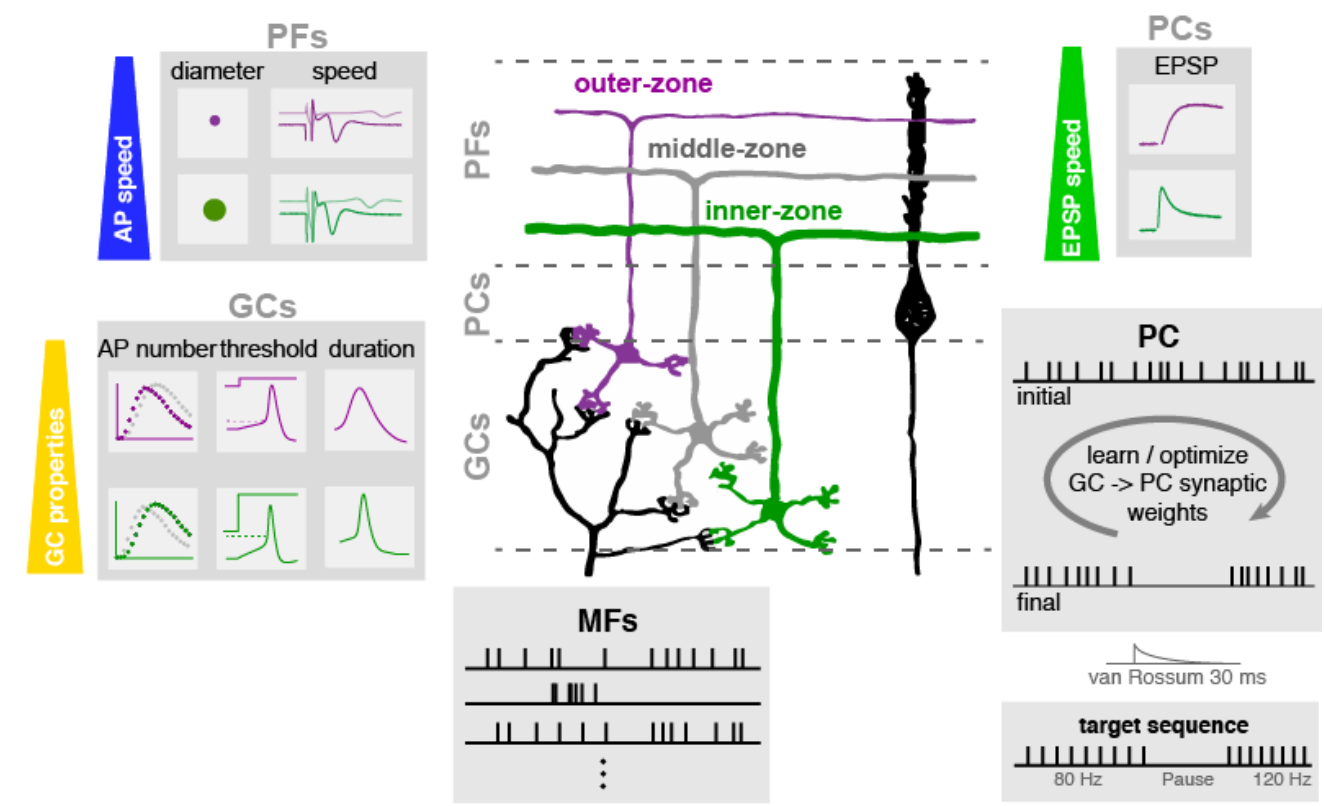

B

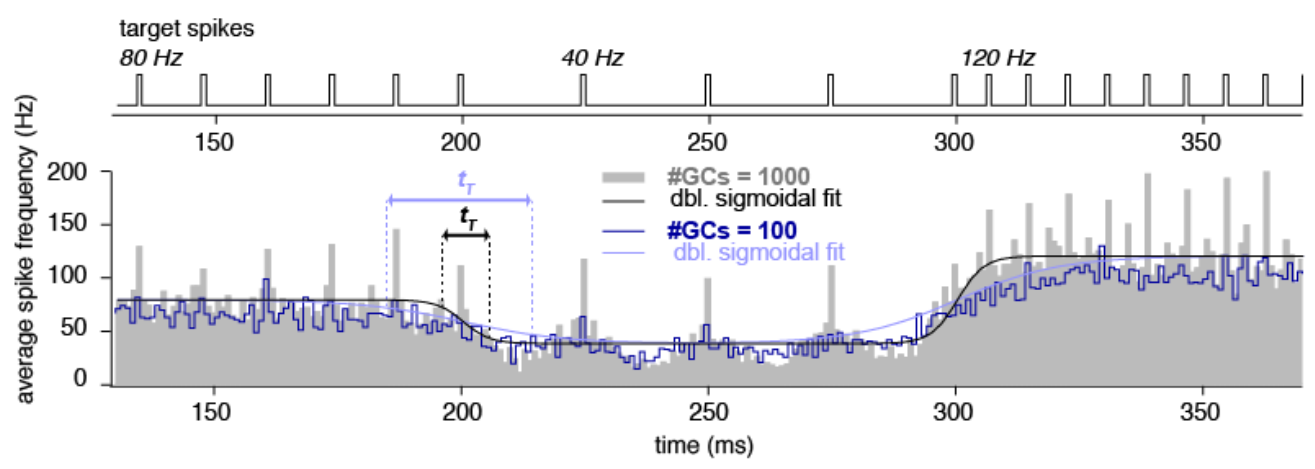

C
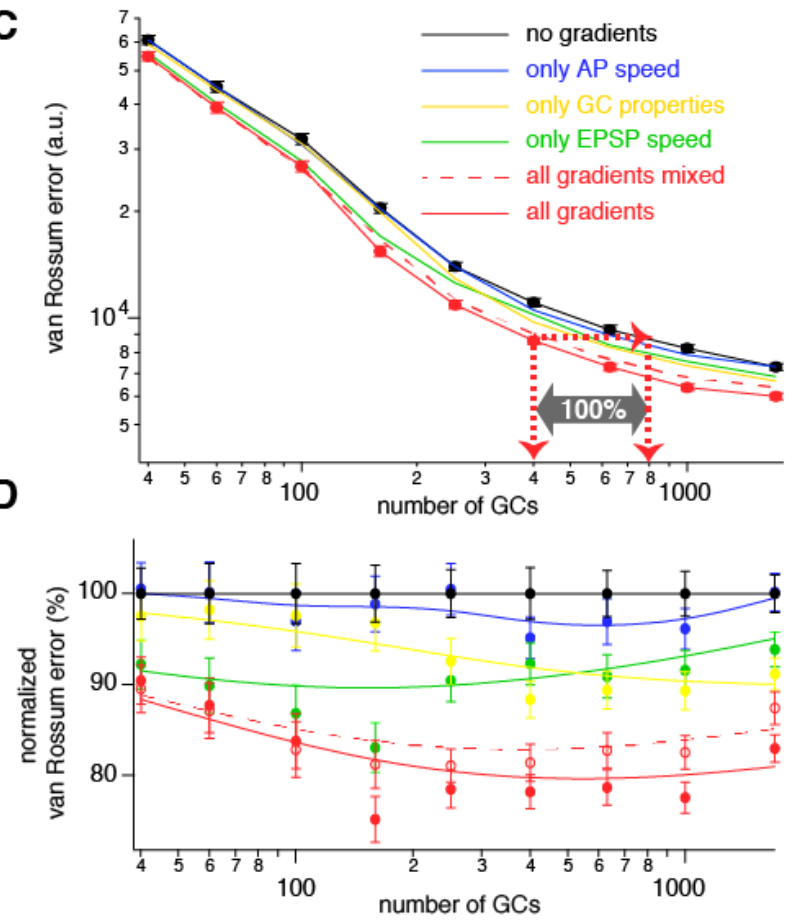

$\mathbf{E}$

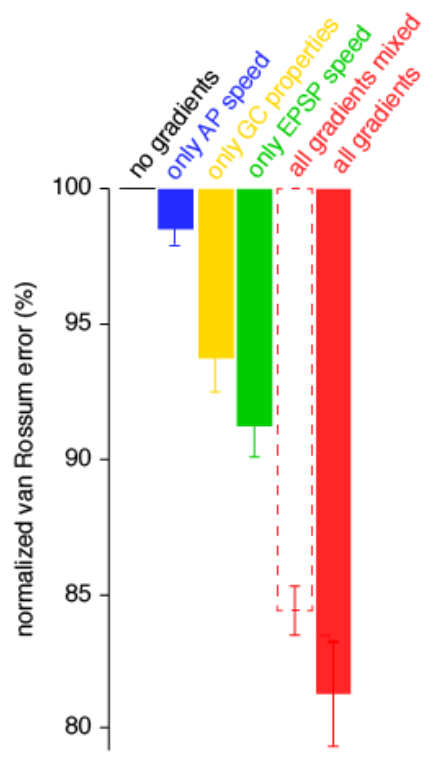


1162 Figure 8: The observed neuronal gradients increase storing capacity and 1163 improve temporal precision of PC spiking

1164 A. Schematic illustration of the network model of the cerebellar cortex as explained in the main text.

B. Average spiking histogram for models consisting of 100 and 1000 GCs, superimposed with double sigmoidal fits constrained to 80,40 and $120 \mathrm{~Hz}$. Above the target spiking sequence is indicated. $t_{T}$ indicates the transition time of the sigmoidal fit for the respective number of GCs.

C. Double logarithmic plot of the average minimal van Rossum error plotted against the number of GCs for a model with no gradients (black), a model with only gradually varied GC parameters (yellow), PF propagation speed (blue), EPSP kinetics (green), and all gradients (red). Furthermore, all parameters were gradually varied but the connectivity between GC, PF and EPSPs was random (all gradients mixed; dashed red). Red dashed lines with arrows indicate the number of GCs needed to obtain the same van Rossum error with all gradients compared to no gradients. With no gradients $100 \%$ more GCs are needed to obtain the same van Rossum error.

D. Average van Rossum errors as shown in panel $\mathrm{C}$ but normalized to the value of the model without gradients, superimposed with a smoothing spline interpolation.

E. Error bar indicate average of the relative differences shown in panel D. 

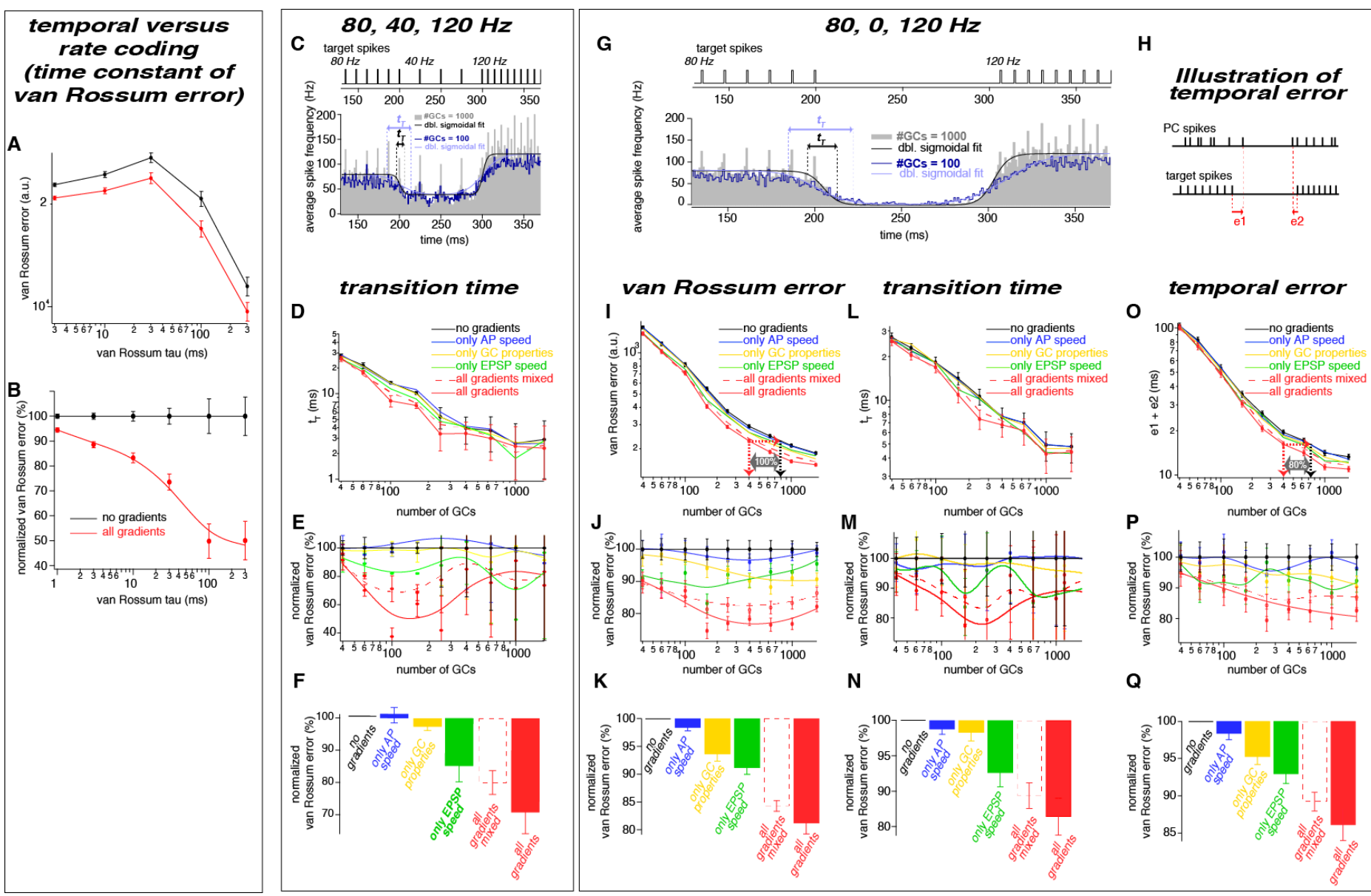

Q
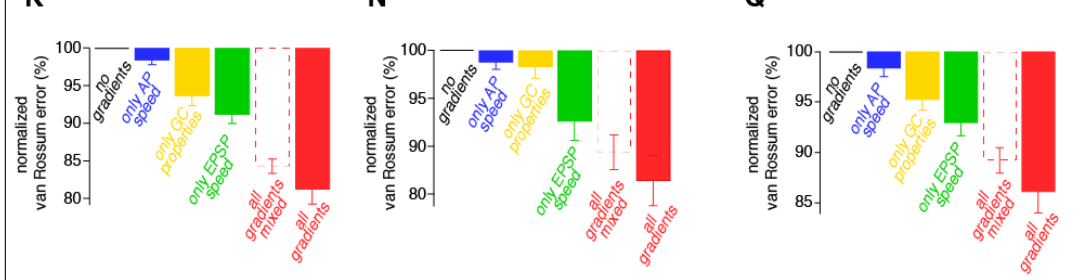

1187 Figure 8-figure supplement 1: The observed neuronal gradients reduce 1188 the temporal error and improve rate coding of PC spikes.

$1189 \quad$ A. Double logarithmic plot of the van Rossum error of model with 300

1190 GCs without (black) and with all gradients (red) plotted versus the time

1191 constant of the van Rossum kernel ranging from 2 to $300 \mathrm{~ms}$.

1192

B. Data as in panel A normalized to the model without gradients.

1193

C. Average spiking histogram for models consisting of 100 and 1000

1194 GCs, superimposed with double sigmoidal fits constrained to 80,40

1195 and $120 \mathrm{~Hz}$. Above the target spiking sequence is indicated.

1196

D. Double logarithmic plot of the transition time $\left(t_{T}\right)$ of the double 1197 exponential fits as illustrated in panel C. Error bars represent $95 \%$ confidence intervals.

1199

E. Transition time $\left(t_{T}\right)$ as shown in panel $D$ but normalized to the value of 1200 the model without gradients, superimposed with a smoothing spline interpolation.

1202

F. Average of the relative differences shown in panel $\mathrm{E}$.

1203

G. Average spiking histogram for models consisting of 100 and 1000 GCs, superimposed with double sigmoidal fits constrained to 80,0 and 
$120 \mathrm{~Hz}$. Above the target spiking sequence with 80,0 , and $120 \mathrm{~Hz}$ is indicated.

$\mathrm{H}$. Illustration of the temporal error (e1 and e2) of the spikes defining the beginning and the end of the pause. I-K, L-N, and O-Q. Same plots as D-F but for the $80,0,120 \mathrm{~Hz}$ target sequence and the van Rossum error $(\mathrm{I}-\mathrm{K})$, the transition time $\left(t_{T}\right)(\mathrm{L}-\mathrm{N})$ and 
A

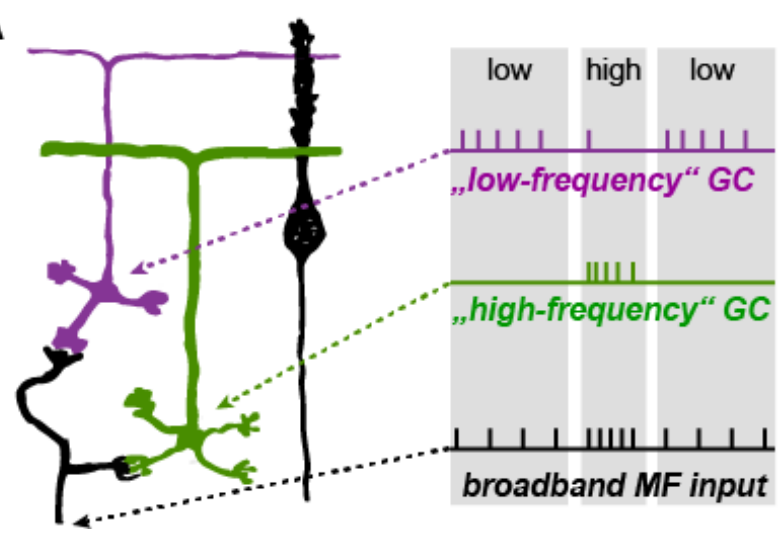

B

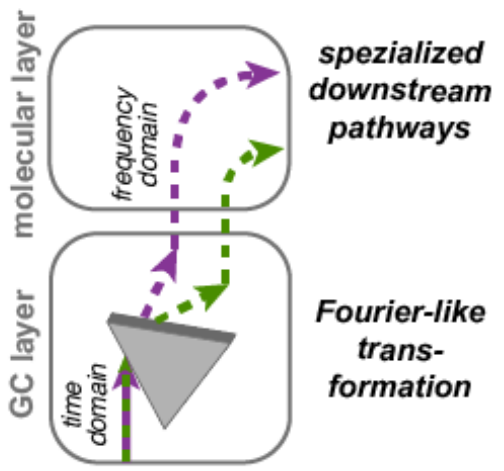

1215 Figure 9: Illustration of the concept of Fourier-like transformation in the 1216 cerebellar cortex

1217

1218

1219

1220

1221

1222

1223

1224

1225

1226

1227
A. Illustration of a broadband MF inputs conveying a sequence of low, high, and low firing frequency. Inner-zone GCs will preferentially fire during high-frequency inputs ('high-frequency' GC) and outer-zone GCs during low-frequency inputs ('low-frequency' GC).

B. Schematic illustration of the signal flow through the cerebellar cortex. The Fourier-like transformation in the GC layer is illustrated as an optical prism separating the spectral components on the MF input. Thereby the MF signal in the time domain is transformed to the frequency domain and sent via specialized signaling pathways in the molecular layer to the PC. 
1228

1229

1230

1231

1232

1233

1234

1235

1236

1237

1238

1239

1240

1241

1242

1243

1244

1245

1246

1247

1248

1249

1250

1251

1252

1253

1254

1255

1256

1257

1258

1259

1260

1261

1262

1263

1264

1265

1266

1267

1268

1269

1270

1271

1272

\section{References}

Albergaria, C., Silva, N.T., Pritchett, D.L., and Carey, M.R. (2018). Locomotor activity modulates associative learning in mouse cerebellum. Nat Neurosci 21, 725-735.

Albus, J.S. (1971). A Theory of Cerebellar Function. Math Biosci 10, 25-61.

Apps, R., Hawkes, R., Aoki, S., Bengtsson, F., Brown, A.M., Chen, G., Ebner, T.J., Isope, P., Jörntell, H., Lackey, E.P., et al. (2018). Cerebellar modules and their role as operational cerebellar processing units. The Cerebellum 17, 654-682.

Arenz, A., Silver, R.A., Schaefer, A.T., and Margrie, T.W. (2008). The contribution of single synapses to sensory representation in vivo. Science 321, 977-980.

Baade, C., Byczkowicz, N., and Hallermann, S. (2016). NMDA receptors amplify mossy fiber synaptic inputs at frequencies up to at least $750 \mathrm{~Hz}$ in cerebellar granule cells. Synapse 70, 269-276.

Babadi, B., and Sompolinsky, H. (2014). Sparseness and expansion in sensory representations. Neuron 83, 1213-1226.

Baginskas, A., Palani, D., Chiu, K., and Raastad, M. (2009). The H-current secures action potential transmission at high frequencies in rat cerebellar parallel fibers. Eur J Neurosci 29, 87-96.

Barbour, B. (1993). Synaptic currents evoked in Purkinje cells by stimulating individual granule cells. Neuron 11, 759-769.

Bengtsson, F., and Jörntell, H. (2009). Sensory transmission in cerebellar granule cells relies on similarly coded mossy fiber inputs. Proc Natl Acad Sci U S A 106, 2389.

Billings, G., Piasini, E., Lőrincz, A., Nusser, Z., and Silver, R.A. (2014). Network structure within the cerebellar input layer enables lossless sparse encoding. Neuron 83, 960-974.

Blot, A., and Barbour, B. (2014). Ultra-rapid axon-axon ephaptic inhibition of cerebellar Purkinje cells by the pinceau. Nat Neurosci 17, 289-295.

Blot, A., de Solages, C., Ostojic, S., Szapiro, G., Hakim, V., and Lena, C. (2016). Timeinvariant feed-forward inhibition of Purkinje cells in the cerebellar cortex in vivo. J Physiol 594, 2729-2749.

Braitenberg, V., Heck, D., and Sultan, F. (1997). The detection and generation of sequences as a key to cerebellar function: Experiments and theory. Behav Brain Sci 20, 229-\&.

Brown, S.T., and Raman, I.M. (2018). Sensorimotor Integration and Amplification of Reflexive Whisking by Well-Timed Spiking in the Cerebellar Corticonuclear Circuit. Neuron 99, 564-575 e562.

Brunel, N., Hakim, V., Isope, P., Nadal, J.P., and Barbour, B. (2004). Optimal information storage and the distribution of synaptic weights: perceptron versus Purkinje cell. Neuron 43, 745-757.

Cayco-Gajic, N.A., Clopath, C., and Silver, R.A. (2017). Sparse synaptic connectivity is required for decorrelation and pattern separation in feedforward networks. Nat Commun 8, 1116.

Cayco-Gajic, N.A., and Silver, R.A. (2019). Re-evaluating Circuit Mechanisms Underlying Pattern Separation. Neuron 101, 584-602. 
1273

1274

1275

1276

1277

1278

1279

1280

1281

1282

1283

1284

1285

1286

1287

1288

1289

1290

1291

1292

1293

1294

1295

1296

1297

1298

1299

1300

1301

1302

1303

1304

1305

1306

1307

1308

1309

1310

1311

1312

1313

1314

1315

1316

1317

1318

Cembrowski, M.S., and Spruston, N. (2019). Heterogeneity within classical cell types is the rule: lessons from hippocampal pyramidal neurons. Nat Rev Neurosci 20, 193-204.

Chabrol, F.P., Arenz, A., Wiechert, M.T., Margrie, T.W., and DiGregorio, D.A. (2015). Synaptic diversity enables temporal coding of coincident multisensory inputs in single neurons. Nat Neurosci 18, 718-727.

Chadderton, P., Margrie, T.W., and Häusser, M. (2004). Integration of quanta in cerebellar granule cells during sensory processing. Nature 428, 856-860.

Clopath, C., and Brunel, N. (2013). Optimal properties of analog perceptrons with excitatory weights. PLoS Comput Biol 9, e1002919.

Clopath, C., Nadal, J.P., and Brunel, N. (2012). Storage of correlated patterns in standard and bistable Purkinje cell models. PLoS Comput Biol 8, e1002448.

D'Angelo, E., Rossi, P., and Taglietti, V. (1993). Different proportions of N-methyl-Daspartate and non-N-methyl-D-aspartate receptor currents at the mossy fibregranule cell synapse of developing rat cerebellum. Neuroscience 53, 121-130.

De Schutter, E., and Bower, J.M. (1994a). An active membrane model of the cerebellar Purkinje cell II. Simulation of synaptic responses. J Neurophysiol 71, 401-419.

De Schutter, E., and Bower, J.M. (1994b). An active membrane model of the cerebellar Purkinje cell. I. Simulation of current clamps in slice. J Neurophysiol 71, 375-400.

De Schutter, E., and Bower, J.M. (1994c). Simulated responses of cerebellar Purkinje cells are independent of the dendritic location of granule cell synaptic inputs. Proc Natl Acad Sci U S A 91, 4736-4740.

Dean, D., Porrill, J., Ekerot, C.F., and Jörntell, H. (2010). The cerebellar microcircuit as an adaptive filter: experimental and computational evidence. Nat Neurosci 11, 30-43.

Delvendahl, I., Straub, I., and Hallermann, S. (2015). Dendritic patch-clamp recordings from cerebellar granule cells demonstrate electrotonic compactness. Front Cell Neurosci 9.

Desai, N.S., Gray, R., and Johnston, D. (2017). A Dynamic Clamp on Every Rig. eNeuro 4.

Dhar, M., Hantman, A.W., and Nishiyama, H. (2018). Developmental pattern and structural factors of dendritic survival in cerebellar granule cells in vivo. Sci Rep $8,17561$.

Dieudonne, S., and Dumoulin, A. (2000). Serotonin-driven long-range inhibitory connections in the cerebellar cortex. J Neurosci 20, 1837-1848.

Dizon, M.J., and Khodakhah, K. (2011). The role of interneurons in shaping purkinje cell responses in the cerebellar cortex. J Neurosci 31, 10463-10473.

Doussau, F., Schmidt, H., Dorgans, K., Valera, A.M., Poulain, B., and Isope, P. (2017). Frequency-dependent mobilization of heterogeneous pools of synaptic vesicles shapes presynaptic plasticity. Elife 6.

du Lac, S., Raymond, J.L., Sejnowski, T.J., and Lisberger, S.G. (1995). Learning and memory in the vestibulo-ocular reflex. Annu Rev Neurosci 18, 409-441.

Eccles, J.C., Ito, M., and Szentagothai, J. (1967). The Cerebellum as a Neuronal Machine (Berlin: Springer-Verlag). 
bioRxiv preprint doi: https://doi.org/10.1101/764886; this version posted September 10, 2019. The copyright holder for this preprint (which was not certified by peer review) is the author/funder, who has granted bioRxiv a license to display the preprint in perpetuity. It is made available under aCC-BY 4.0 International license.

\section{4}

1319

1320

1321

1322

1323

1324

1325

1326

1327

1328

1329

1330

1331

1332

1333

1334

1335

1336

1337

1338

1339

1340

1341

1342

1343

1344

1345

1346

1347

1348

1349

1350

1351

1352

1353

1354

1355

1356

1357

1358

1359

1360

1361

1362

1363

Espinosa, J.S., and Luo, L. (2008). Timing neurogenesis and differentiation: insights from quantitative clonal analyses of cerebellar granule cells. J Neurosci 28, 23012312.

Fletcher, L.N., and Williams, S.R. (2019). Neocortical Topology Governs the Dendritic Integrative Capacity of Layer 5 Pyramidal Neurons. Neuron 101, 76-90 e74.

Fox, C.A., and Barnard, J.W. (1957). A quantitative study of the Purkinje cell dendritic branchlets and their relationship to afferent fibres. J Anat 91, 299-313.

Fujita, M. (1982). Adaptive filter model of the cerebellum. Biol Cybern 45, 195-206.

Gaffield, M.A., and Christie, J.M. (2017). Movement Rate Is Encoded and Influenced by Widespread, Coherent Activity of Cerebellar Molecular Layer Interneurons. J Neurosci 37, 4751-4765.

Galliano, E., Gao, Z., Schonewille, M., Todorov, B., Simons, E., Pop, Andreea S., D’Angelo, E., van den Maagdenberg, Arn M.J.M., Hoebeek, Freek E., and De Zeeuw, Chris I. (2013). Silencing the Majority of Cerebellar Granule Cells Uncovers Their Essential Role in Motor Learning and Consolidation. Cell Reports 3, 1239-1251.

Gao, Z., Proietti-Onori, M., Lin, Z., Ten Brinke, M.M., Boele, H.J., Potters, J.W., Ruigrok, T.J., Hoebeek, F.E., and De Zeeuw, C.I. (2016). Excitatory Cerebellar Nucleocortical Circuit Provides Internal Amplification during Associative Conditioning. Neuron 89, 645-657.

Gao, Z., van Beugen, B.J., and De Zeeuw, C.I. (2012). Distributed synergistic plasticity and cerebellar learning. Nat Rev Neurosci 13, 619-635.

Garrido, J.A., Ros, E., and D'Angelo, E. (2013). Spike timing regulation on the millisecond scale by distributed synaptic plasticity at the cerebellum input stage: a simulation study. Front Comput Neurosci 7, 64.

Gibo, T.L., Criscimagna-Hemminger, S.E., Okamura, A.M., and Bastian, A.J. (2013). Cerebellar motor learning: are environment dynamics more important than error size? J Neurophysiol 110, 322-333.

Giovannucci, A., Badura, A., Deverett, B., Najafi, F., Pereira, T.D., Gao, Z., Ozden, I., Kloth, A.D., Pnevmatikakis, E., Paninski, L., et al. (2017). Cerebellar granule cells acquire a widespread predictive feedback signal during motor learning. Nat Neurosci 20, 727-734.

Guo, C., Witter, L., Rudolph, S., Elliott, H.L., Ennis, K.A., and Regehr, W.G. (2016). Purkinje Cells directly inhibit granule cells in specialized regions of the cerebellar cortex. Neuron 91, 1330-1341.

Hallermann, S., Fejtova, A., Schmidt, H., Weyhersmüller, A., Silver, R.A., Gundelfinger, E., and Eilers, J. (2010). Bassoon speeds vesicle reloading at a central excitatory synapse. Neuron $18,710-723$.

Harvey, R.J., and Napper, R.M. (1991). Quantitative studies on the mammalian cerebellum. Prog Neurobiol 36, 437-463.

Heath, N.C., Rizwan, A.P., Engbers, J.D., Anderson, D., Zamponi, G.W., and Turner, R.W. (2014). The expression pattern of a Cav3-Kv4 complex differentially regulates spike output in cerebellar granule cells. J Neurosci 34, 8800-8812.

Heiney, S.A., Wohl, M.P., Chettih, S.N., Ruffolo, L.I., and Medina, J.F. (2014). Cerebellar-dependent expression of motor learning during eyeblink conditioning in head-fixed mice. J Neurosci 34, 14845-14853. 
1364 Henneman, E., Somjen, G., and Carpenter, D.0. (1965). Excitability and inhibitability of motoneurons of different sizes. J Neurophysiol 28, 599-620.

Herculano-Houzel, S. (2009). The human brain in numbers: a linearly scaled-up primate brain. Front Hum Neurosci 3, 31.

Herzfeld, D.J., Kojima, Y., Soetedjo, R., and Shadmehr, R. (2015). Encoding of action by the Purkinje cells of the cerebellum. Nature 526, 439-442.

Hewitt, A.L., Popa, L.S., Pasalar, S., Hendrix, C.M., and Ebner, T.J. (2011). Representation of limb kinematics in Purkinje cell simple spike discharge is conserved across multiple tasks. J Neurophysiol 106, 2232-2247.

1373

1374

1375

1376

1377

1378

1379

1380

1381

Hong, S., Negrello, M., Junker, M., Smilgin, A., Thier, P., and De Schutter, E. (2016). Multiplexed coding by cerebellar Purkinje neurons. Elife 5.

Houghton, C., and Kreuz, T. (2012). On the efficient calculation of van Rossum distances. Network 23, 48-58.

Howarth, C., Gleeson, P., and Attwell, D. (2012). Updated energy budgets for neural computation in the neocortex and cerebellum. J Cereb Blood Flow Metab 32, 1222-1232.

1382

1383

1384

1385

1386

1387

1388

1389

Isler, K., and van Schaik, C.P. (2006). Metabolic costs of brain size evolution. Biol Lett 2, 557-560.

Isope, P., and Barbour, B. (2002). Properties of unitary granule cell-->Purkinje cell synapses in adult rat cerebellar slices. J Neurosci 22, 9668-9678.

Jack, J.J.B., Noble, D., and Tsien, R.W. (1983). Electric current flow in excitable cells. (Oxford: Clarendon Press).

Jayant, N., Johnston, J., and Safranek, R. (1993). Signal compression based on models of human perception. Proceedings of the IEEE 81, 1385 - 1422.

Jörntell, H., and Ekerot, C.F. (2006). Properties of somatosensory synaptic integration in cerebellar granule cells in vivo. J Neurosci 26, 11786-11797.

1390

1391

1392

1393

1394

1395

1396

1397

1398

1399

1400

1401

Kan, P.L.v., Gibson, A.R., and Houk, J.C. (1993). Movement-related inputs to intermediate cerebellum of the monkey. J Neurophysiol 69, 74-94.

Keele, S.W., and Ivry, R. (1990). Does the cerebellum provide a common computation for diverse tasks? A timing hypothesis. Ann N Y Acad Sci 608, 179207.

Krieger, C., Shinoda, Y., and Smith, A.M. (1985). Labelling of cerebellar mossy fiber afferents with intra-axonal horseradish peroxidase. Exp Brain Res 59, 414-417.

Lackey, E.P., Heck, D.H., and Sillitoe, R.V. (2018). Recent advances in understanding the mechanisms of cerebellar granule cell development and function and their contribution to behavior. F1000Res 7.

Llinás, R., and Sugimori, M. (1980). Electrophysiological properties of in vitro Purkinje cell dendrites in mammalian cerebellar slices. J Physiol 305, 197-213.

1402

1403

1404

1405

Longair, M.H., Baker, D.A., and Armstrong, J.D. (2011). Simple Neurite Tracer: open source software for reconstruction, visualization and analysis of neuronal processes. Bioinformatics 27, 2453-2454.

1406

1407

Marr, D. (1969). A theory of cerebellar cortex. J Physiol (Lond) 202, 437-470.

Martin, T.A., Keating, J.G., Goodkin, H.P., Bastian, A.J., and Thach, W.T. (1996). Throwing while looking through prisms. I. Focal olivocerebellar lesions impair 1408 adaptation. Brain 119 1183-1198. 
bioRxiv preprint doi: https://doi.org/10.1101/764886; this version posted September 10, 2019. The copyright holder for this preprint (which was not certified by peer review) is the author/funder, who has granted bioRxiv a license to display the preprint in perpetuity. It is made available under aCC-BY 4.0 International license.

\section{6}

1409

1410

1411

1412

1413

1414

1415

1416

1417

1418

1419

1420

1421

1422

1423

1424

1425

1426

1427

1428

1429

1430

1431

1432

1433

1434

1435

1436

1437

1438

1439

1440

1441

1442

1443

1444

1445

1446

1447

1448

1449

1450

1451

1452
Masoli, S., Solinas, S., and D'Angelo, E. (2015). Action potential processing in a detailed Purkinje cell model reveals a critical role for axonal compartmentalization. Front Cell Neurosci 9, 47.

Mauk, M.D., and Buonomano, D.V. (2004). The neural basis of temporal processing. Annu Rev Neurosci 27, 307-340.

Medina, J.F., Garcia, K.S., Nores, W.L., Taylor, N.M., and Mauk, M.D. (2000). Timing mechanisms in the cerebellum: testing predictions of a large-scale computer simulation. J Neurosci 20, 5516-5525.

Medina, J.F., and Lisberger, S.G. (2007). Variation, signal, and noise in cerebellar sensory-motor processing for smooth-pursuit eye movements. J Neurosci 27, 6832-6842.

Mittmann, W., Koch, U., and Häusser, M. (2005). Feed-forward inhibition shapes the spike output of cerebellar Purkinje cells. J Physiol (Lond) 563, 369-378.

Palay, S.M., and Chan-Palay, V. (1974). Cerebellar cortex: cytology and organization (Springer, Berlin).

Palkovits, M., Magyar, P., and Szentagothai, J. (1971). Quantitative histological analysis of the cerebellar cortex in the cat. 3 . Structural organization of the molecular layer. Brain Res 34, 1-18.

Payne, H.L., French, R.L., Guo, C.C., Nguyen-Vu, T.B., Manninen, T., and Raymond, J.L. (2019). Cerebellar Purkinje cells control eye movements with a rapid rate code that is invariant to spike irregularity. Elife 8.

Pichitpornchai, C., Rawson, J.A., and Rees, S. (1994). Morphology of parallel fibres in the cerebellar cortex of the rat: an experimental light and electron microscopic study with biocytin. J Comp Neurol 342, 206-220.

Pike, F.G., Goddard, R.S., Suckling, J.M., Ganter, P., Kasthuri, N., and Paulsen, O. (2000). Distinct frequency preferences of different types of rat hippocampal neurones in response to oscillatory input currents. J Physiol 529 Pt 1, 205-213.

Rakic, P. (1972). Extrinsic cytological determinants of basket and stellate cell dendritic pattern in the cerebellar molecular layer. J Comp Neurol 146, 335-354.

Raman, I.M., and Bean, B.P. (1997). Resurgent sodium current and action potential formation in dissociated cerebellar Purkinje neurons. J Neurosci 17, 4517-4526.

Ramón y Cajal, S. (1911). Histologie du Système Nerveux de l'Homme et des Vertébrés. (A. Maloine, Paris).

Rancz, E.A., Ishikawa, T., Duguid, I., Chadderton, P., Mahon, S., and Häusser, M. (2007). High-fidelity transmission of sensory information by single cerebellar mossy fibre boutons. Nature 450, 1245-1248.

Raymond, J.L., and Medina, J.F. (2018). Computational Principles of Supervised Learning in the Cerebellum. Annu Rev Neurosci 41, 233-253.

Ritzau-Jost, A., Delvendahl, I., Rings, A., Byczkowicz, N., Harada, H., Shigemoto, R., Hirrlinger, J., Eilers, J., and Hallermann, S. (2014). Ultrafast action potentials mediate kilohertz signaling at a central synapse. Neuron 84, 152-163.

Rizwan, A.P., Zhan, X., Zamponi, G.W., and Turner, R.W. (2016). Long-Term Potentiation at the Mossy Fiber-Granule Cell Relay Invokes Postsynaptic SecondMessenger Regulation of Kv4 Channels. J Neurosci 36, 11196-11207. 
bioRxiv preprint doi: https://doi.org/10.1101/764886; this version posted September 10, 2019. The copyright holder for this preprint (which was not certified by peer review) is the author/funder, who has granted bioRxiv a license to display the preprint in perpetuity. It is made available under aCC-BY 4.0 International license.

\section{7}

1453

1454

1455

1456

1457

1458

1459

1460

1461

1462

1463

1464

1465

1466

1467

1468

1469

1470

1471

1472

1473

1474

1475

1476

1477

1478

1479

1480

1481

1482

1483

1484

1485

1486

1487

1488

1489

1490

1491

1492

1493

1494

1495

1496

Rossert, C., Dean, P., and Porrill, J. (2015). At the Edge of Chaos: How Cerebellar Granular Layer Network Dynamics Can Provide the Basis for Temporal Filters. PLoS Comput Biol 11, e1004515.

Rossum, M.C.W.v. (2001). A Novel Spike Distance. Neural Comput 13, 751-763.

Roth, A., and Häusser, M. (2001). Compartmental models of rat cerebellar Purkinje cells based on simultaneous somatic and dendritic patch-clamp recordings. J Physiol 535, 445-472.

Sarnaik, R., and Raman, I.M. (2018). Control of voluntary and optogenetically perturbed locomotion by spike rate and timing of neurons of the mouse cerebellar nuclei. Elife 7.

Saviane, C., and Silver, R.A. (2006). Fast vesicle reloading and a large pool sustain high bandwidth transmission at a central synapse. Nature 439, 983-987.

Serôdio, P., and Rudy, B. (1998). Differential Expression of Kv4 K+Channel Subunits Mediating Subthreshold Transient K+ (A-Type) Currents in Rat Brain. J Neurophysiol 79, 1081-1091.

Silver, R.A., Traynelis, S.F., and Cull-Candy, S.G. (1992). Rapid-time-course miniature and evoked excitatory currents at cerebellar synapses in situ. Nature 355, 163166.

Singla, S., Dempsey, C., Warren, R., Enikolopov, A.G., and Sawtell, N.B. (2017). A cerebellum-like circuit in the auditory system cancels responses to self-generated sounds. Nat Neurosci 20, 943-950.

Soltesz, I., and Losonczy, A. (2018). CA1 pyramidal cell diversity enabling parallel information processing in the hippocampus. Nat Neurosci 21, 484-493.

Spanne, A., and Jörntell, H. (2013). Processing of multi-dimensional sensorimotor information in the spinal and cerebellar neuronal circuitry: a new hypothesis. PLoS Comput Biol 9, e1002979.

Steuber, V., Mittmann, W., Hoebeek, F.E., Silver, R.A., De Zeeuw, C.I., Häusser, M., and De Schutter, E. (2007). Cerebellar LTD and pattern recognition by Purkinje cells. Neuron 54, 121-136.

Sudhakar, S.K., Hong, S., Raikov, I., Publio, R., Lang, C., Close, T., Guo, D., Negrello, M., and De Schutter, E. (2017). Spatiotemporal network coding of physiological mossy fiber inputs by the cerebellar granular layer. PLoS Comput Biol 13, e1005754.

Sultan, F., and Bower, J.M. (1998). Quantitative Golgi study of the rat cerebellar molecular layer interneurons using principal component analysis. J Comp Neurol 393, 353-373.

Suvrathan, A., Payne, H.L., and Raymond, J.L. (2016). Timing rules for synaptic plasticity matched to behavioral function. Neuron 92, 959-967.

Tsodyks, M.V., and Markram, H. (1997). The neural code between neocortical pyramidal neurons depends on neurotransmitter release probability. Proc Natl Acad Sci U S A 94, 719-723.

Valera, A.M., Binda, F., Pawlowski, S.A., Dupont, J.L., Casella, J.F., Rothstein, J.D., Poulain, B., and Isope, P. (2016). Stereotyped spatial patterns of functional synaptic connectivity in the cerebellar cortex. Elife 5. 
1497

1498

1499

1500

1501

1502

1503

1504

1505

1506

1507

1508

1509

1510

1511

1512

1513

1514

1515

1516

1517

1518

1519

1520

1521

1522

1523

1524

1525

1526

1527

1528

1529

1530

1531

1532

1533

1534

1535

1536

1537

Valera, A.M., Doussau, F., Poulain, B., Barbour, B., and Isope, P. (2012). Adaptation of granule cell to purkinje cell synapses to high-frequency transmission. J Neurosci 32, 3267-3280.

van Kan, P.L., Gibson, A.R., and Houk, J.C. (1993). Movement-related inputs to intermediate cerebellum of the monkey. J Neurophysiol 69, 74-94.

van Rossum, M.C. (2001). A novel spike distance. Neural Comput 13, 751-763.

Vranesic, I., Iijima, T., Ichikawa, M., Matsumoto, G., and Knöpfel, T. (1994). Signal transmission in the parallel fiber-Purkinje cell system visualized by highresolution imaging. Proc Natl Acad Sci U S A 91, 13014-13017.

Wadiche, J.I., and Jahr, C.E. (2005). Patterned expression of Purkinje cell glutamate transporters controls synaptic plasticity. Nat Neurosci 8, 1329-1334.

Wagner, M.J., Kim, T.H., Savall, J., Schnitzer, M.J., and Luo, L. (2017). Cerebellar granule cells encode the expectation of reward. Nature 544, 96-100.

Wallace, G.K. (1992). The JPEG still picture compression standard. IEEE Transactions on Consumer Electronics 38, xviii - xxxiv.

Walter, J.T., and Khodakhah, K. (2009). The advantages of linear information processing for cerebellar computation. Proc Natl Acad Sci U S A 106, 4471-4476.

Wang, W.C., and Brehm, P. (2017). A Gradient in Synaptic Strength and Plasticity among Motoneurons Provides a Peripheral Mechanism for Locomotor Control. Curr Biol 27, 415-422.

Williams, R.W., and Herrup, K. (1988). The control of neuron number. Annu Rev Neurosci 11, 423-453.

Wilms, C.D., and Häusser, M. (2015). Reading out a spatiotemporal population code by imaging neighbouring parallel fibre axons in vivo. Nat Commun 6, 6464.

Witter, L., Canto, C.B., Hoogland, T.M., de Gruijl, J.R., and De Zeeuw, C.I. (2013). Strength and timing of motor responses mediated by rebound firing in the cerebellar nuclei after Purkinje cell activation. Front Neural Circuits 7, 133.

Witter, L., and De Zeeuw, C.I. (2015a). In Vivo Differences in Inputs and Spiking Between Neurons in Lobules VI/VII of Neocerebellum and Lobule $\mathrm{X}$ of Archaeocerebellum. Cerebellum 14.

Witter, L., and De Zeeuw, C.I. (2015b). Regional functionality of the cerebellum. Curr Opin Neurobiol 33, 150-155.

Xiao, J., Cerminara, N.L., Kotsurovskyy, Y., Aoki, H., Burroughs, A., Wise, A.K., Luo, Y., Marshall, S.P., Sugihara, I., Apps, R., and Lang, E.J. (2014). Systematic regional variations in Purkinje cell spiking patterns. PLoS ONE 9, e105633.

Yamazaki, T., and Tanaka, S. (2007). A spiking network model for passage-of-time representation in the cerebellum. Eur J Neurosci 26, 2279-2292.

Zhou, H.B., Lin, Z.M., Voges, K., Ju, C.H., Gao, Z.Y., Bosman, L.W.J., Ruigrok, T.J., Hoebeek, F.E., De Zeeuw, C.I., and Schonewille, M. (2014). Cerebellar modules operate at different frequencies. Elife 3. 